\title{
Absorption, Metabolism, and Excretion, In Vitro Pharmacology, and Clinical Pharmacokinetics of Ozanimod, a Novel Sphingosine 1-Phosphate Receptor Modulator ${ }^{\text {\} }$
}

\author{
Sekhar Surapaneni, Usha Yerramilli, April Bai, Deepak Dalvie, Jennifer Brooks, Xiaomin Wang, \\ Julie V. Selkirk, Yingzhuo Grace Yan, Peijin Zhang, Richard Hargreaves, Gondi Kumar, \\ Maria Palmisano, and Jonathan Q. Tran
}

Non-clinical Research and Development, Bristol Myers Squibb, Summit, New Jersey (S.S., U.Y., D.D., X.W., G.K.); Drug Metabolism and Pharmacokinetics, Escient Pharmaceuticals, San Diego, California (J.B.); Neuroscience TRC, Bristol Myers Squibb, Princeton, New Jersey (J.V.S., Y.G.Y., R.H.); and Clinical Pharmacology and Pharmacometrics and Research and Early Development, Bristol Myers Squibb, Summit, New Jersey (P.Z., M.P., J.Q.T.)

Received August 21, 2020; accepted January 26, 2021

\begin{abstract}
Ozanimod is approved for the treatment of relapsing forms of multiple sclerosis. Absorption, metabolism, and excretion of ozanimod were investigated after a single oral dose of $1.0 \mathrm{mg}$ $\left[{ }^{14} \mathrm{C}\right]$ ozanimod hydrochloride to six healthy subjects. In vitro experiments were conducted to understand the metabolic pathways and enzymes involved in the metabolism of ozanimod and its active metabolites. The total mean recovery of the administered radioactivity was $\sim 63 \%$, with $\sim 26 \%$ and $\sim 37 \%$ recovered from urine and feces, respectively. Based on exposure, the major circulating components were active metabolite $\mathrm{CC} 112273$ and inactive metabolite RP101124, which together accounted for $50 \%$ of the circulating total radioactivity exposure, whereas ozanimod accounted for $6.7 \%$ of the total radioactive exposure. Ozanimod was extensively metabolized, with 14 metabolites identified, including two major active metabolites (CC112273 and CC1084037) and one major inactive metabolite (RP101124) in circulation. Ozanimod is metabolized by three primary pathways, including aldehyde dehydrogenase and alcohol dehydrogenase, cytochrome P450 isoforms 3A4 and
\end{abstract}

1A1, and reductive metabolism by gut microflora. The primary metabolite RP101075 is further metabolized to form major active metabolite CC112273 by monoamine oxidase $B$, which further undergoes reduction by carbonyl reductases to form $\mathrm{CC} 1084037$ or CYP2C8-mediated oxidation to form RP101509. CC1084037 is oxidized rapidly to form CC112273 by aldo-keto reductase $1 \mathrm{C1} / 1 \mathrm{C} 2$ and/or $3 \beta$ - and $11 \beta$ hydroxysteroid dehydrogenase, and this reversible oxidoreduction between two active metabolites favors CC112273. The ozanimod example illustrates the need for conducting timely radiolabeled human absorption, distribution, metabolism, and excretion studies for characterization of disproportionate metabolites and assessment of exposure coverage during drug development.

\section{SIGNIFICANCE STATEMENT}

Absorption, metabolism, and excretion of ozanimod were characterized in humans, and the enzymes involved in complex metabolism were elucidated. Disproportionate metabolites were identified, and the activity of these metabolites was determined.
There was no external funding for this work.

All authors except Jennifer Brooks and Jonathan Q. Tran are current employees and shareholders of Bristol Myers Squibb (formerly Celgene), and Jennifer Brooks and Jonathan Q. Tran were former employees of Celgene when the work described in this manuscript was conducted.

https://doi.org/10.1124/dmd.120.000220.

S This article has supplemental material available at dmd.aspetjournals.org.

\section{Introduction}

Ozanimod is a sphingosine 1-phosphate (S1P) receptor modulator that binds with high affinity selectively to $\mathrm{S} 1 \mathrm{P}$ receptor subtypes $1\left(\mathrm{~S}_{1} \mathrm{P}_{1}\right)$ and $5\left(\mathrm{~S}_{1} \mathrm{P}_{5}\right)$. The $\mathrm{S} 1 \mathrm{P}_{1}$ receptor is expressed by lymphocytes, dendritic cells, cardiomyocytes, and vascular endothelial cells and is involved in the regulation of chronic inflammation (via mediation of lymphocyte movement), heart rate, smooth muscle tone, and endothelial function (Subei and Cohen, 2015; Brinkmann et al., 2002; Brinkmann, 2009; Karuppuchamy et al., 2017). Ozanimod acts as a functional antagonist of the $\mathrm{S}_{1} \mathrm{P}_{1}$ by promoting sustained receptor internalization, resulting in

ABBREVIATIONS: ADH, alcohol dehydrogenase; ADME, absorption, distribution, metabolism, and excretion; AE, adverse event; AKR, aldo-keto reductase; ALDH, aldehyde dehydrogenase; AME, absorption, metabolism, and excretion; AMS, accelerator mass spectrometry; AUC, area under the plasma concentration-time curve; $\mathrm{AUC}_{\infty}$, area under the curve from time 0 extrapolated to infinity; $\mathrm{AUC}_{\text {last }}$, area under the curve from time 0 to last measurable concentration; CBR, carbonyl reductases; FA, formic acid; GTPyS, guanosine 5'-O-[gamma-thio]triphosphate; HCl, hydrochloride; HLM, human liver microsome; HPLC, high performance liquid chromatography; HSD, hydroxysteroid dehydrogenase; IS, internal standard; $\mathrm{K}_{2} \mathrm{EDTA}$, dipotassium EDTA; LC-MS, liquid chromatography and mass spectrometry; LC-MS/MS, liquid chromatography tandem mass spectrometry; LSC, liquid scintillation counter; MAO, monoamine oxidase; MS/MS, tandem mass spectrometry; NAT, $N$-acetyltransferase; PK, pharmacokinetics; rCYP, recombinant cytochrome P450 enzyme; rCYP3A4, recombinant cytochrome 3A4; S1P, sphingosine 1-phosphate; $t_{1 / 2}$, half-life; $\mathrm{T}_{\text {max }}$, time to maximum concentration; TNO, Toegepast Natuurwetenschappelijk Onderzoek; UPLC, ultra-performance liquid chromatography. 
a reduction of the number of circulating lymphocytes (Scott et al., 2016). Ozanimod also demonstrates activity at the $\mathrm{S}_{1} \mathrm{P}_{5}$, which supports oligodendrocyte progenitor process extension and survival and contributes to blood-brain barrier integrity (Miron et al., 2008; van Doorn et al., 2012). Ozanimod is approved in the United States for the treatment of adults with relapsing forms of multiple sclerosis and in European Union for the treatment of adults with relapsing-remitting multiple sclerosis. The mechanism by which ozanimod exerts therapeutic effects in MS is unknown but may involve the reduction of lymphocyte migration into the central nervous system. Ozanimod is also in clinical development for the treatment of moderate to severe ulcerative colitis and Crohn disease (Sandborn et al., 2016; Feagan et al., 2020).

Early metabolism studies in animals identified three pharmacologically active metabolites (RP101988, RP101075, and RP101442) that have similar S1P selectivity and potency in vitro to ozanimod (Scott et al., 2013). The pharmacokinetics (PK) of ozanimod, RP101988, RP101075, and RP101442 in healthy subjects has been published previously (Tran et al., 2017, 2018a,b). However, the absorption, metabolism, and excretion (AME) study is important during the clinical development, as it helps to identify and quantify circulating parent and metabolites and elucidate the elimination pathways of the medicinal product. Results from the AME study can be used to evaluate the potential contribution of any metabolites to the overall safety and/or efficacy profile of the drug and the potential risk for drug-drug interactions (Coppola et al., 2019). As per the FDA guidance on safety testing of drug metabolites, metabolites identified only in human plasma or metabolites present at disproportionately higher levels in humans than in any of the animal test species $(>10 \%$ of total drug-related exposure at steady state) should be considered for safety assessment (FDA, 2020). Here, we report results from three evaluations: 1) the human AME study in healthy male subjects after a single $1-\mathrm{mg}$ oral dose of $\left[{ }^{14} \mathrm{C}\right]$ ozanimod hydrochloride, 2) in vitro metabolism and identification of human metabolic enzymes involved in the metabolism of ozanimod, and 3) in vitro $\mathrm{S} 1 \mathrm{P}$ receptor activity profile of metabolites.

\section{Materials and Methods}

\section{Study Drug}

An oral solution of $\left[{ }^{14} \mathrm{C}\right]$ ozanimod hydrochloride $(\mathrm{HCl})$ formulated at a dose strength of $1 \mathrm{mg}$ with approximately $37 \mu \mathrm{Ci}(1.3 \mathrm{MBq})$ was manufactured by Quotient Clinical (Nottingham, United Kingdom). The structure of ozanimod and position of the radiolabel is shown in Fig. 1.

\section{Clinical Study Design and Subjects}

This was an open-label, single-dose study in healthy male subjects (clinicaltrials.gov ID: NCT02994381). The clinical study was performed by Quotient Clinical in accordance with the principles of the Declaration of Helsinki and International Council for Harmonization Good Clinical Practice Guidelines approved by the Committee for Medicinal Products for Human Use (1996, updated 2002). The study protocol and informed consent form were approved by

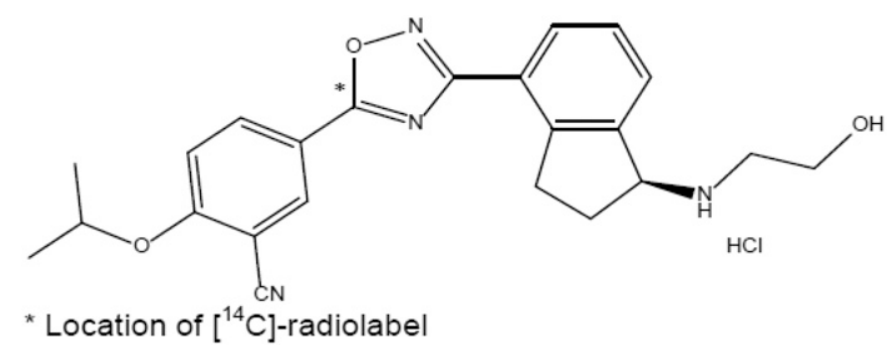

Fig. 1. Structure of ozanimod, with the site of the ${ }^{14} \mathrm{C}$ label indicated $(*)$. the ethics committee of the study center (Wales Research Ethics Committee 2, Cardiff, Wales). All subjects provided written informed consent to participate in the study.

Subjects were screened for eligibility to participate in the study up to 28 days before dosing. Six healthy male subjects who were nonsmokers, aged 30-65 years, and had a body mass index ranging from 18.0 to $32.0 \mathrm{~kg} /$ $\mathrm{m}^{2}$ were enrolled in the study. Subjects were of good health as determined by past medical history, physical examination, vital signs, ECG, and laboratory tests. The subjects also had no history of alcoholism or drug abuse and did not use any prescription drug, over-the-counter drug (except for paracetamol at $\leq 2$ g per day), or herbal remedies within 14 days (28 days for St. John's wort) before dosing and had no radiation exposure exceeding $5 \mathrm{mSv}$ in the last 12 months or $10 \mathrm{mSv}$ in the last 5 years.

Subjects were admitted to the clinical study unit 1 day prior to dosing (day -1 ). Subjects received a single oral dose of $1 \mathrm{mg}$ of $\left[{ }^{14} \mathrm{C}\right]$ ozanimod hydrochloride (equivalent to $0.92 \mathrm{mg}$ of ozanimod) on the morning of day 1 after a standard breakfast. The formulation consisted of $5 \% \mathrm{w} / \mathrm{v}$ hydroxypropyl- $\beta$-cyclodextrin solution at a concentration of $0.1 \mathrm{mg} / \mathrm{ml}$ of $\left[{ }^{14} \mathrm{C}\right]$ ozanimod hydrochloride. Blood samples, urine, and feces were collected up to 168 hours after dosing with ozanimod. Subjects remained residents in the clinical study unit until 168 hours after dosing and were discharged on day 8. Further collections (up to 240 hours postdose) of urine and feces (up to 504 hours postdose) were obtained at home, as subjects had not achieved a mass balance cumulative recovery of $>90 \%$ or $<1 \%$ of the dose administered collected in urine and feces within two separate consecutive 24-hour periods by day 8 .

Physical examinations, 12-lead ECGs, vital sign measurements, and clinical laboratory tests were performed, and adverse events (AEs) and concomitant medications were monitored throughout the study to assess safety and tolerability.

\section{Sample Collection}

After ozanimod dosing, blood samples were collected at predose ( 0 hour) and $1,2,3,4,6,8,12,24,36,48,72,96,120,144$, and 168 hours for analysis of total radioactivity in whole blood and plasma, PK of ozanimod and metabolites in plasma, and metabolic profiling in plasma. For analysis of total radioactivity in whole blood, venous blood samples were collected into 2-ml dipotassium EDTA ( $\mathrm{K}_{2}$ EDTA) tubes and placed immediately onto crushed ice. Samples were frozen within 30 minutes of collection at $-70^{\circ} \mathrm{C}$ or below until they were shipped to Toegepast Natuurwetenschappelijk Onderzoek (TNO) (Utrechtseweg, Netherlands) for the analysis of total radioactivity.

For analysis of plasma total radioactivity, venous blood samples were collected into 4-ml $\mathrm{K}_{2}$ EDTA tubes and processed to plasma. The resultant plasma was transferred into polypropylene tubes $(2 \times 3.5 \mathrm{ml}$; primary and backup; each tube was to contain approximately $1 \mathrm{ml}$ of plasma). Plasma samples were frozen within 1 hour of collection at $-70^{\circ} \mathrm{C}$ or below until they were shipped to TNO for the analysis of total radioactivity. For PK analysis of ozanimod and metabolites in plasma, blood samples were collected into 6- $\mathrm{ml} \mathrm{K}_{2}$ EDTA tubes and processed to plasma. The resultant plasma was transferred into a 3.5-ml polypropylene tube (primary) and mixed gently, and half the volume was transferred into another appropriately labeled 3.5-ml polypropylene tube (backup). The primary tube was to contain $1 \mathrm{ml}$ of plasma. Plasma samples were frozen within 1 hour of collection at $-70^{\circ} \mathrm{C}$ or below until they were shipped to ICON Laboratory Services for the analysis of ozanimod and its metabolites. For metabolite profiling and identification, venous blood samples were collected into $\mathrm{K}_{2}$ EDTA tubes $(2 \times$ $10 \mathrm{ml}$ ) and placed immediately onto crushed ice. Samples were centrifuged at $2800 \mathrm{rpm}$ for 15 minutes at $4^{\circ} \mathrm{C}$ within 20 minutes of collection. The resultant plasma was aliquoted into two appropriately labeled polypropylene tubes $(3.5-\mathrm{ml}$ tube for primary and 10-ml tube for backup). The primary tube was to contain $1 \mathrm{ml}$ of plasma, and the backup was to contain $7 \mathrm{ml}$ of plasma. Plasma samples were frozen within 1 hour of collection at $-70^{\circ} \mathrm{C}$ or below until they were shipped to TNO for metabolite profiling and identification.

Urine samples were collected at the following intervals: predose (within 1 hour before dosing); 0-6, 6-12, 12-24 hours; and then every 24 hours until 240 hours postdose. Urine was collected into individual Triton-coated polyethylene containers. After sample collection, the weight of each individual sample was recorded, and the sample was stored at $2-8^{\circ} \mathrm{C}$ until they were shipped daily to Pharmaron for analysis of total radioactivity. The urine samples, after extraction from pooled samples, were shipped at $-70^{\circ} \mathrm{C}$ from Pharmaron to TNO for metabolite profiling and identification. 
Feces were collected at the following intervals: admission to predose and every 24 hours until 504 hours postdose. Toilet papers were retained from day 1 until discharge. Fecal samples were collected into individual polypropylene containers. After sample collection, the weight of each individual sample was recorded, and the samples were stored at approximately $-20^{\circ} \mathrm{C}$ until they were shipped daily to Pharmaron for analysis of total radioactivity. The fecal samples, after extraction from pooled samples, were shipped at $-70^{\circ} \mathrm{C}$ for metabolite profiling and identification.

\section{Total Radioactivity Measurement}

Radioactivity in urine and dose bottle rinses was quantified directly by using a liquid scintillation counter (LSC) with automatic external standard quench correction. Samples were mixed with scintillant (Ultima Gold XR) and counted (2300TR, 2900TR, or 3100TR Tri-Carb, Scintillation Counter; Perkin Elmer). Duplicate aliquots of $5 \mathrm{ml}$ of urine were directly mixed with scintillant and counted for determination of radioactivity. Radioactivity in fecal homogenate was determined after combustion in oxygen using an Automatic Sample Oxidizer (model 307; Perkin Elmer). Duplicate weighed samples of fecal homogenates $(0.1-0.5 \mathrm{~g})$ were combusted, and the combustion products were absorbed into CarboSorb and mixed with the scintillator cocktail PermaFluor E+ for measurement of radioactivity. The limit of quantification using LSC was taken as twice the background dpm value for samples of the same type. Resulting limit of quantification values were 0.06 (urine) and 0.75 (feces) ng equivalents per gram.

Radioactivity levels were low for plasma and blood matrices; therefore, total radioactivity was determined in plasma and whole-blood samples by measuring the carbon-14 using accelerator mass spectrometry (AMS) and converting the ${ }^{14} \mathrm{C} /{ }^{12} \mathrm{C}$ isotope ratio to units of radioactivity. A reference standard with a certified ${ }^{14} \mathrm{C} /{ }^{12} \mathrm{C}$ isotope ratio was used for suitability samples and analyzed first before samples. Five standard samples were included in each batch analysis with a minimum of three replicates. The lower limit of quantification for plasma and whole-blood samples was 0.58 and $1.70 \mathrm{pg}$ eq $/ \mathrm{ml}$, respectively.

\section{Determination of Plasma Concentrations of Ozanimod and Metabolites}

Plasma concentrations of ozanimod and its metabolites CC112273, RP101988, RP101075, RP101124, and RP101442 were determined using validated analytical methods. In addition, urine concentrations of ozanimod were determined using validated analytical methods. Briefly, aliquots of 100-200 $\mu$ l plasma were mixed with $25 \mu \mathrm{l}$ internal standards and extracted by support-liquid extraction (Isolute LSE+; Biotage, Charlotte, NC), and $200 \mu$ l reconstituted sample was subjected to LC-MS/MS analysis. The LC-MS/MS system consisted of a reversed-phase ultra-high-pressure liquid chromatography (Kinetex C18, $100 \times 3.0$ $\mathrm{mm}, 2.6 \mu \mathrm{m}$; Phenomenex, Torrance, CA) with electrospray tandem mass spectrometry (MS/MS) detection (API Sciex 6500 QTRAP; AB Sciex, Framingham, MA). The methods were validated over the concentration ranges of 4-2000 $\mathrm{pg} / \mathrm{ml}$ for ozanimod and RP101075, 16-4000 pg/ml for RP101988, 8.00-4000 $\mathrm{pg} / \mathrm{ml}$ for RP101442 and RP101124, and 25-10,000 pg/ml for CC112273, respectively.

Similarly, aliquots of $500 \mu 1$ (Triton-treated) urine were mixed with $50 \mu 1$ internal standards and extracted by liquid-liquid extraction (methyl tert-butyl ether), and $150 \mu \mathrm{l}$ reconstituted sample was subjected to LC-MS/MS analysis. The methods were validated over the concentration $4.00-2000 \mathrm{pg} / \mathrm{ml}$ for both RPC1063 and RP101988 and 16.0-4000 pg/ml for RP101075, respectively.

\section{Determination of Metabolite Profiles in Plasma and Excreta}

Urine and homogenate fecal metabolite profiling were conducted by high performance liquid chromatography (HPLC) fraction collection and off-line LSC. Fractions were collected approximately every 14.9 seconds for the duration of the analytical run using a CTC HTX-xt Pal fraction collector. Fractions were collected into four 96-well deep-well LumaPlates-96. The 96-well plates were evaporated to dryness in a sample evaporator (HT-4X or DD-4X; Genevac). Radioactivity (as counts per minute) was counted off-line using a Packard TopCount NXT microplate scintillation and luminescence counter. Overall extraction recovery for urine and feces was greater than $87 \%$ and $72 \%$, respectively.

Because of the low levels of radioactivity in plasma, the profiling was conducted by using HPLC fraction collection and AMS. Metabolite profiling and identification of ozanimod metabolites in plasma, urine, and feces was performed by TNO and Pharmaron. Plasma samples were pooled and extracted with 3 times the volume of acetonitrile. Six individual plasma sample pools were prepared for 0 - to 96 -hour samples by using the Hamilton pooling method. Approximately $3 \mathrm{ml}$ of supernatant was transferred to a tube containing $150 \mu \mathrm{l}$ of DMSO and evaporated until 150-200 $\mu \mathrm{l}$ was remaining. Approximately $450 \mu \mathrm{l}$ of ammonium acetate $\mathrm{pH} 8$ buffer was added and mixed thoroughly. The plasma extraction efficiency is greater than $90 \%$ across six individual subjects. This was subjected to ultra-performance liquid chromatography (UPLC) separation, and fractionations were collected every 6-10 seconds using Collect PAL autosampler (LEAP Technologies, Raleigh, NC). The HPLC column was a Waters BEH $2.1 \times 5 \mathrm{~mm}$ i.d. with $1.7-\mu \mathrm{m}$ particle size, and the mobile phases consisted of $20 \mathrm{mM}$ ammonium acetate ( $\mathrm{pH} 8.0)$ as mobile phase $\mathrm{A}$ and methanol as mobile phase $\mathrm{B}$. The initial composition was $80 \%$ mobile phase A and changed to $20 \%$ over a 30-minute gradient. The collected fractions were subjected to AMS measurements using a SSAMS-250 system (National Electrostatics Corporation, Middleton, WI). The remaining fractions were used for metabolite identification by mass spectrometry (Q-Exactive Plus; ThermoScientific, UK, or API-5500; AB Sciex, UK). In addition, representative blank human plasma, urine, and fecal extracts were spiked with authentic standards of ozanimod and its metabolites and analyzed by HPLC-UV detection to match the retention times of analytes in samples.

Metabolite profiling and identification of ozanimod metabolites in plasma, urine, and feces was performed by TNO and Pharmaron. Accelerator mass spectrometry was used when appropriate.

\section{Pharmacokinetic Evaluation}

Pharmacokinetic analyses of plasma concentration-time data and radioactivitytime data were performed using noncompartmental analysis by Phoenix WinNonlin version 6.3 (Certara, Inc.). The following PK parameters were estimated: maximum observed concentration $\left(\mathrm{C}_{\mathrm{max}}\right)$, area under the curve from time 0 to last measurable concentration $\left(\mathrm{AUC}_{\text {last }}\right)$, area under the curve from time 0 extrapolated to infinity $\left(\mathrm{AUC}_{\infty}\right)$, apparent elimination half-life $\left(t_{1 / 2}\right)$, apparent total clearance, and apparent volume of distribution.

\section{Structural Characterization of Metabolites}

Metabolite identification using accurate mass full scan and product ion analyses was carried out on selected human plasma, urine, and feces samples to screen for the presence of previously characterized or known metabolites. Samples were searched for metabolites corresponding to the supplied reference standards; potential hydroxylated (or $\mathrm{N}$-oxide), $\mathrm{N}$-/O-dealkylated, ketone, or acid metabolites; and/or conjugated metabolites. Samples were also screened for components formed by oxadiazole ring opening and/or cleavage, together with potential resulting oxidative deaminated and/or acid metabolites. In total, 18 reference standards of the metabolites were synthesized and made available for metabolite identification or quantitation in plasma (Martinborough et al., 2015; Tran et al., 2017, 2020). Full-scan techniques were used to attempt to identify any additional unassigned components by direct comparison with the ${ }^{14} \mathrm{C}$ radiochromatogram. A combination of high-resolution mass spectrometry or LC-MS/MS techniques were employed to characterize the low abundant metabolites with use of authentic reference standards. The liquid chromatography and mass spectrometry (LC-MS) system consisted of Q-Exactive in electrospray ionization mode with both positive and negative polarity (ThermoFisher Scientific). LC-MS/MS with multiple reaction monitoring-based analysis was carried out using a triple quadrupole AB Sciex QTrap 5500 (AB Sciex) instrument in electro spray ionization (ESI) positive ionization mode.

\section{In Vitro Metabolism}

Human Liver Microsomes, Hepatocytes, and Recombinant Human Cytochrome P450 Enzymes. In vitro metabolism experiments with ozanimod or its metabolites were evaluated using pooled mixed-sex liver fractions ( $\mathrm{S} 9$ and microsomes) and hepatocytes. In vitro experiments with microsomes prepared from cells expressing recombinant cytochromes P450 (1A1,1A2, 2C9, 2C19, $2 \mathrm{D} 6,3 \mathrm{~A} 4)$ were conducted with the same methodology as the liver microsome experiments utilizing $100 \mathrm{pmol} / \mathrm{ml}$ recombinant cytochromes P450 (final concentration). Hepatocyte incubations were conducted with 1 million cells per milliliter and incubated for 1-2 hours with periodic sampling. Control substrates and test compounds were incubated at 0.25 or $1 \mu \mathrm{M}$ concentrations. 
In Vitro Experiments with Chemical Inhibitors. In vitro studies were conducted to identify enzymes responsible for $N$-dealkylation and carboxylation using recombinant human cytochromes $\mathrm{P} 450$ and other oxidative enzymes and to assess the effect of chemical inhibitors of cytochrome P450 isozymes as well as non-cytochrome P450 enzymes. Chemical inhibitors furafylline (1A2), quercetin (2C8), sulfaphenazole (2C9), quinidine (2D6), ketoconazole (3A4 plus others), oxybutynin (CYP2C19), raloxifene (aldehyde oxidase), clomethiazole (2E1), ticlopidine (2C19/2B6), disulfiram (aldehyde dehydrogenase), and 4-methylpyrazole (alcohol dehydrogenase) were used as selective inhibitors of respective enzymes. Typically, incubations were performed with $0.1 \mathrm{M}$ phosphate buffer, $\mathrm{pH} 7.4$, at $37^{\circ} \mathrm{C}$ containing microsomal protein $(0.5-1 \mathrm{mg} / \mathrm{ml}$ or recombinant enzyme $100 \mathrm{pmol} / \mathrm{ml}$ of each cytochrome P450 enzyme) with or without chemical inhibitors in a volume of $0.2-0.5$ $\mathrm{ml}$. The reactions were started by addition of $10 \mathrm{mM}$ NADPH (final $1 \mathrm{mM}$ ), and the controls had buffer instead of NADPH. For experiments in which the role of dehydrogenases was investigated (formation of RP101988 from ozanimod), experiments were conducted with human liver microsomes in the presence of both NAD+ and NADPH. Dehydrogenases are commonly more active at higher $\mathrm{pH}$ and use NAD+ as a cofactor instead of NADPH; hence, the formation of RP101988 from ozanimod in liver microsomes or S9 in the presence or absence of $\mathrm{NAD+}$ or NADPH at $\mathrm{pH} 7.4$ or 8.5 was also tested. All incubations were conducted for an optimized time of 60 minutes, unless stated otherwise.

Investigation of Formation of CC112273 and Its Downstream Metabolites. To identify the enzyme catalyzing the formation of CC112273, ozanimod or RP101075 was incubated with human liver microsomes, liver S9, mitochondria, or cytosol or with recombinant enzymes monoamine oxidase (MAO) A or B in the presence and absence of NADPH. The reaction mixture containing phosphate buffer (100 mM, pH 7.4), NADPH (1 mM), and human liver microsomes (0.5 mg/ $\mathrm{ml}$ ) was preincubated for 3 minutes. Control experiments (experiments without NADPH) were conducted by replacing NADPH with phosphate buffer. Incubations were started by addition of RP101075 $(1 \mu \mathrm{M})$. The final incubation volume was $0.5 \mathrm{ml}$. After the addition of RP101075, 100- $\mu$ l aliquots were removed from each incubation at 0 minutes and added to $200 \mu 1$ acetonitrile containing $0.2 \mu \mathrm{M}$ deuterated (d5) 7-ethoxycoumarin [internal standard (IS)]. The remaining reaction mixtures were incubated at $37^{\circ} \mathrm{C}$ in a shaking water bath for 60 minutes, during which the reaction was established to be linear. At 60 minutes, another aliquot of $100 \mu \mathrm{l}$ was removed and added to $200 \mu \mathrm{l}$ acetonitrile containing $0.2 \mu \mathrm{M}$ IS. The quenched aliquots were vortexed for 5 minutes and centrifuged (approximately $4000 \mathrm{~g}$, room temperature for 10 minutes) to obtain the supernatant, which was analyzed for CC112273 and CC1084037 by LC-MS/MS. The effects of concentration, cofactor, or chemical inhibitors on the formation of CC112273, RP112289, and CC1084037 were determined.

Recombinant human MAO-A and MAO-B expressed in baculovirus-infected insect cells were obtained from Sigma-Aldrich, Saint Louis, Missouri. Reaction mixtures containing phosphate buffer (100 mM; pH 7.4) with $\mathrm{MgCl}_{2}$ (5 mM), recombinant MAO-A, or MAO-B $(0.025 \mathrm{mg} / \mathrm{ml})$ were preincubated for 3 minutes. Reaction mixtures containing phosphate buffer (100 mM; pH 7.4) with $\mathrm{MgCl}_{2}$ (5 mM), recombinant MAO-A and MAO-B $(0.025 \mathrm{mg} / \mathrm{ml})$, chlorgyline, and deprenyl $(0.5 \mu \mathrm{M}$ each) were preincubated for 10 minutes. The reaction was started with the addition of RP101075 or ozanimod $(1.0 \mu \mathrm{M})$ and incubated at $37^{\circ}$ $\mathrm{C}$ in a shaking water bath for 180 minutes. The final incubation volume was $1 \mathrm{ml}$. Aliquots $(100 \mu \mathrm{l})$ of the reaction mixture were taken at $0,60,120$, and 180 minutes and added to $200 \mu \mathrm{l}$ acetonitrile containing $0.2 \mu \mathrm{M}$ IS [0.2 $\mu \mathrm{M}$ deuterated (d5) 7-thoxycoumarin] to stop the reaction. The reaction mixture was centrifuged (approximately $4000 \mathrm{~g}$, room temperature, 10 minutes) to obtain the supernatant, which was analyzed for CC112273 by LC-MS/MS. All incubations were performed in triplicate.

In Vitro Metabolism of CC1084037. To determine the metabolic stability of CC1084037, CC1084037 was incubated with human hepatocytes, microsomes, and cytosol at $5 \mu \mathrm{M}$. Stability of CC1084037 in human hepatocytes was determined by incubating CC1084037 at $5 \mu \mathrm{M}$ with $1.0 \times 106$ million cells per milliliter human hepatocytes, and aliquots of the incubation mixture were sampled at 0-, 5-, 10-, 15-, 30-, 45-, and 60-minute time intervals.

To determine the metabolic stability of CC1084037 in human liver subcellular fractions, $\mathrm{CC} 1084037$ at $5 \mu \mathrm{M}$ was incubated with pooled human liver microsomes, cytosol, and $\mathrm{S} 9$ at $0.25 \mathrm{mg} / \mathrm{ml}$ in phosphate buffer $(100 \mathrm{mM}, \mathrm{pH}$ 7.4) containing $\mathrm{MgCl}_{2}(8 \mathrm{mM})$. Reactions were initiated by the addition of $2 \mathrm{mM}$ $\mathrm{NADP}+$ or NAD+ and allowed to proceed for various times (5-60 minutes). The reaction was quenched with equal volume of $0.1 \%$ formic acid (FA) in acetonitrile (v/v) containing CC1084037-d3 as internal standard at $50 \mathrm{ng} / \mathrm{ml}$. After centrifugation, the supernatant was analyzed for RP112273 by LC-MS/MS. All the incubations were carried out in triplicate.

To identify the enzymes responsible for the metabolism of CC1084037, CC1084037 was incubated with commercially available recombinant oxidoreductive enzymes from the families of aldo-keto reductase (AKR), alcohol dehydrogenase $(\mathrm{ADH})$, and carbonyl reductase $(\mathrm{CBR})$. The recombinant enzymes used for the study were ADH1B, ADH1C, CBR1, CBR3, and CBR4 and AKRs 1A1, 1B1, 1B10, 1C1, 1C2, 1C3, 1C4, 1D1, 7A2, and 7A3. Incubation mixture (100- $\mu$ l final) was prepared by mixing potassium phosphate buffer $(100 \mathrm{mM}, \mathrm{pH}$ 7.4), $\mathrm{MgCl}_{2}$ (5 mM), CC1084037 (5 $\left.\mu \mathrm{M}\right)$, and recombinant enzyme (10 $\left.\mu \mathrm{g} / \mathrm{ml}\right)$ in a tapered 96-well plate. The reaction mixture was preincubated for 5 minutes in a shaking water bath at $37^{\circ} \mathrm{C}$. The reaction was initiated by adding $2 \mathrm{mM}$ NADP+ and incubated in a shaking water bath for 60 minutes at $37^{\circ} \mathrm{C}$. The reaction was quenched by adding one volume of acetonitrile in $0.1 \%$ FA containing CC1084037-d3 (50 ng/ml) internal standard. The quenched reaction mixture was vortexed and centrifuged at $4000 \mathrm{rpm}$ (2950g) for 10 minutes, and the supernatant was injected $(4 \mu \mathrm{l})$ into the LC-MS for analysis. All incubations were performed in triplicate.

Inhibition studies with chemical inhibitors phenolphthalein (AKR 1C family inhibitor), menadione (CBR inhibitor), glycyrrhetinic acid (11 $\beta$-HSD inhibitor), and trilostane ( $3 \beta$-HSD) were performed by mixing phosphate buffer $(100 \mathrm{mM}$, $\mathrm{pH}$ 7.4), $\mathrm{MgCl}_{2}$ (8 mM), HLM, human liver mitochondria, human liver cytosol, or S9 $(0.25 \mathrm{mg} / \mathrm{ml})$ with CC1084037 $(5 \mu \mathrm{M})$ and chemical inhibitor $(20$ or $100 \mu \mathrm{M})$. The reaction mixture was preincubated in a shaking water bath for 5 minutes at $37^{\circ} \mathrm{C}$. The reaction was initiated by adding cofactor $2 \mathrm{mM} \mathrm{NADP}+$ or NAD+ and was incubated in a shaking water bath at $37^{\circ} \mathrm{C}$ for 30 minutes. DMSO was added in control reactions instead of inhibitor. At the end of the incubation time, the reactions were quenched by adding one volume of acetonitrile in $0.1 \%$ FA containing IS (CC1084037-d3, $50 \mathrm{ng} / \mathrm{ml}$ ). The mixture was vortexed and centrifuged at $4000 \mathrm{rpm}$ (790g) for 10 minutes, and supernatant was subjected to LC-MS/MS analysis. All incubations were performed in triplicate.

Characterization of Anaerobic Metabolism of Ozanimod in Fecal Cultures. In vitro incubations with fecal cultures under anaerobic conditions were conducted to characterize the reductive metabolism of the 1,2,4-oxadiazole ring that is common in ozanimod and its major active metabolites CC112273 and CC1084037. In addition, the loss of ${ }^{14} \mathrm{CO}_{2}$ from major inactive metabolites via decarboxylation pathway was also characterized to understand the low recovery in human mass balance study. In rat, fecal homogenates were prepared and incubated with either ozanimod alone or with $15 \mathrm{mg} / \mathrm{ml}$ of bacitracin, neomycin, and streptomycin for 24 hours prior to introduction of ozanimod or metabolites. Reduction of methylene blue was used as a measure of bacterial activity. The aliquot of samples was analyzed over 24 hours. The LC-MS/MS analysis was conducted by using API4000 QTRAP (ABScience) with Agilent 1200 binary pump system with gradient analysis (mobile phase A, $0.1 \%$ formic acid in water; mobile phase B, $0.1 \%$ formic acid in acetonitrile) on a Luna C8 $20 \mathrm{~cm} \times 2 \mathrm{~mm}$ i.d. 2- $\mu \mathrm{m}$ particle size column.

Characterization of Anaerobic Decarboxylation of Ozanimod Metabolite and Loss of ${ }^{14} \mathrm{CO}_{2}$. Human fecal homogenate $(3-5 \mathrm{ml})$ in duplicate containing $20 \mathrm{mM}$ glucose and $\left[{ }^{14} \mathrm{C}\right] \mathrm{RP} 112533$ were purged with nitrogen, the tubes were placed in a plastic pouch with AnaerobGen Compact paper sachet, and the pouch was sealed immediately with a sealing clip. Plastic bags were incubated at $37^{\circ} \mathrm{C}$ for 96 hours in a water bath. To confirm the formation of decarboxylated metabolite of RP112533 by fecal bacteria under anaerobic conditions, spiked homogenate was incubated in the presence of $1 \mathrm{mg} / \mathrm{ml}$ penicillin and streptomycin. Also, $\left[{ }^{14} \mathrm{C}\right]$ carbon dioxide released during the incubation was trapped in Carbo-Sorb E. After the incubation, an aliquot $(100-300 \mu \mathrm{l})$ of incubate was extracted with three volumes of methanol containing IS (RP105846) at $100 \mathrm{ng} / \mathrm{ml}$, and samples were centrifuged. Supernatant was diluted with $0.1 \%$ formic acid in water and injected onto LC-MS/MS. Analyte peak to IS (RP105846) area ratios of RP112533 and 2-hydroxybenzonitrile (decarboxylated metabolite of RP112533) were determined. To determine the loss of total radioactivity due to release of $\left[{ }^{14} \mathrm{C}\right] \mathrm{CO}_{2}$, an aliquot $(\sim 50 \mathrm{mg})$ of spiked homogenate at 0 minutes and 96 hours was combusted in an oxidizer. The LC-MS/MS analysis was conducted for the formation of 2-hydroxy benzonitrile using multiple reaction monitoring in negative ionization mode. The LC-MS/MS system consisted of Shimadzu LCpumps with API4000 QTRAP (ABScience) MS system with gradient analysis 
(mobile phase A, $0.1 \%$ formic acid in water; mobile phase B, $0.1 \%$ formic acid in acetonitrile) on a Phenomenex Kinetics C18 $10 \mathrm{~cm} \times 2 \mathrm{~mm}$ i.d. $2.6-\mu \mathrm{m}$ particle size column.

\section{S1P Receptor Profile and In Vitro Activity Studies}

Membrane Preparation. Membranes were prepared from stable Chinese

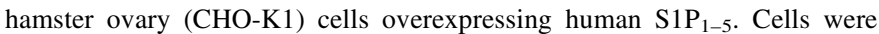
lifted from cell culture trays with $10 \mathrm{mM}$ HEPES, $154 \mathrm{mM} \mathrm{NaCl}$, and $6.85 \mathrm{mM}$ EDTA, pH 7.4. Cells were then pelleted by centrifugation, resuspended, and homogenized in membrane preparation buffer $(10 \mathrm{mM}$ HEPES and $10 \mathrm{mM}$ EDTA, pH 7.4) using a Polytron PT 1200E homogenizer (Kinematica, Luzern, Switzerland) and subsequently centrifuged at $48,000 \mathrm{~g}$ at $4{ }^{\circ} \mathrm{C}$ for 30 minutes in Sorvall RC-6 Ultracentrifuge (Beckman Coulter, Fullerton, CA). The supernatant was removed, and the pellet was rehomogenized and recentrifuged as described above in membrane prep buffer. The final pellet was suspended in ice-cold $10 \mathrm{mM}$ HEPES and $0.1 \mathrm{mM}$ EDTA, pH 7.4, and 1-mg aliquots were prepared and stored at $-80^{\circ} \mathrm{C}$.

$\left[{ }^{35} \mathrm{~S}\right] \mathbf{G T P} \boldsymbol{\gamma} \mathbf{S}$ Binding Assays. $\left[{ }^{35} \mathrm{~S}\right]$ guanosine 5'-O-[gamma-thio]triphosphate $(\mathrm{GTP} \gamma \mathrm{S})$ binding assays were performed in 96-well nonbinding surface plates (Corning) in a final volume of $200 \mu$ l. Test compounds were serial-diluted with DMSO and added to the plates using the Tecan D300E digital printer to total volume of $0.4 \mu \mathrm{l}$ per well. Serial dilution of the endogenous ligand S1P was performed in assay buffer (20 mM HEPES, $10 \mathrm{mM} \mathrm{MgCl}_{2}, 100 \mathrm{mM} \mathrm{NaCl}$, and $1 \mathrm{mM}$ EDTA, pH 7.4, $0.1 \%$ fatty acid-free bovine serum albumin, and $30 \mu \mathrm{g} / \mathrm{ml}$ saponin) and transferred to wells containing the same $0.4 \mu$ l DMSO to keep the DMSO concentration consistent across the entire plate at a final concentration of $0.02 \%$. All wells were then loaded to a total volume of $40 \mu \mathrm{l}$ of assay buffer, with the exception of the wells used to define the nonspecific binding, which received $40 \mu \mathrm{l}$ of $50 \mu \mathrm{M}$ unlabeled GTP $\gamma \mathrm{S}$ (Sigma-Aldrich). CHO-S1P ${ }_{1-5}$ membranes were then added to the plate in a volume of $120 \mu$ l per well of assay buffer containing $40 \mu \mathrm{g} / \mathrm{ml} \mathrm{S} 1 \mathrm{P}$ receptor membrane protein and $2.5 \mathrm{mg} / \mathrm{ml}$ of wheat germ agglutinin-coated polyvinyltoluene scintillation proximity assay beads (PerkinElmer). Also contained in the $120 \mu \mathrm{l}$ per well membrane/assay buffer solution was 5-50 $\mu \mathrm{M}$ GDP (Sigma-Aldrich), specifically $5 \mu \mathrm{M}$ for $\mathrm{S}_{2} \mathrm{P}_{2}$ and $\mathrm{S}_{1} \mathrm{P}_{4}, 16.67 \mu \mathrm{M}$ for $\mathrm{S}_{1} \mathrm{P}_{1}$ and $\mathrm{S}_{1} \mathrm{P}_{5}$, and $50 \mu \mathrm{M}$ for $\mathrm{S}_{1} \mathrm{P}_{3}$. The assay plates were then sealed and incubated at room temperature with gentle agitation for 30 minutes. After 30 minutes of incubation, the radiolabeled $\left[{ }^{35} \mathrm{~S}\right] \mathrm{GTP} \gamma \mathrm{S}$ (PerkinElmer) was added to each well in a volume of $40 \mu \mathrm{l}$ per well of $1 \mathrm{nM}$ of basic buffer (20 mM HEPES, 10 sterile $\mathrm{mM} \mathrm{MgCl}_{2}, 100 \mathrm{mM} \mathrm{NaCl}$, and $1 \mathrm{mM}$ EDTA, pH 7.4) to a final concentration of $200 \mathrm{pM}$. Plates were resealed and incubated at room temperature with gentle agitation for a further 40 minutes. The reaction was then terminated by centrifugation of the plates at $1000 \mathrm{rpm}$ for 3 minutes before the radioactivity bound to the membranes was quantitated by a Perkin Elmer MicroBeta2 2450 microplate scintillation counter.

Data Analysis. The $\left[{ }^{35} \mathrm{~S}\right] \mathrm{GTP} \gamma \mathrm{S}$ binding data after correction for nonspecific binding were normalized to the percent response of the internal S1P control, which was taken to be $100 \%$ for the maximal S1P response and $0 \%$ for the S1P baseline response, and concentration-response curves were generated using nonlinear regression using GraphPad Prism (version 7.03). The potency of the test compounds was reported as $\mathrm{EC}_{50}$ values (concentration of a drug that gives half-maximal response), and the intrinsic activity was reported as the percent maximal response relative to $\mathrm{S} 1 \mathrm{P}$. When a test compound did not elicit a conclusive dose-response curve with a clearly defined maximal response, the $\mathrm{EC}_{50}$ was reported as greater than the highest dose (if less than $50 \%$ ) was or second/third highest dose (if greater than $50 \%$ ) of the test compound.

\section{Results}

\section{Demographic, Safety, and Tolerability Data}

A total of six healthy male subjects enrolled and completed the study. All subjects were Caucasian and had a mean age of 40.3 years (range: 31-63 years), weight of $79.1 \mathrm{~kg}$ (range: $64.6-89.8 \mathrm{~kg}$ ), and body mass index of $26.1 \mathrm{~kg} / \mathrm{m}^{2}$ (range: $22.4-30.1 \mathrm{~kg} / \mathrm{m}^{2}$ ).

A single oral dose of $1 \mathrm{mg}\left[{ }^{14} \mathrm{C}\right]$ ozanimod $\mathrm{HCl}$ was safe and well tolerated. No deaths, serious AEs, or treatment-emergent AEs were reported. There were no clinically significant findings in any laboratory evaluations, vital sign assessments, ECGs, or physical examinations.

\section{Excretion and Mass Balance of Radioactivity in Urine and Feces}

After a single oral dose of $1 \mathrm{mg}\left[{ }^{14} \mathrm{C}\right]$ ozanimod $\mathrm{HCl}$, the total mean recovery of the administered radioactivity by the end of the sampling period (240 hours for urine and 504 hours for feces) was $63 \%$, with $26 \%$ recovered from the urine and $37 \%$ recovered from the feces. Within the first 24 hours postdose, $5.22 \%$ and $0.06 \%$ of the total radioactivity were recovered in the urine and feces, respectively. By 7 days postdose, $<1 \%$ of the total radioactivity was recovered in urine over two consecutive days. By 10 days postdose, $<1 \%$ of the total radioactivity was recovered in feces over two consecutive days, and excretion continued at levels below $1 \%$ daily, and after day 14 postdose below $0.5 \%$ daily, until collections were ceased on day 21 postdose (Fig. 2).

\section{Pharmacokinetics of Total Radioactivity and Ozanimod and Metabolites}

After a single oral dose of $1 \mathrm{mg}\left[{ }^{14} \mathrm{C}\right]$ ozanimod $\mathrm{HCl}$, the total radioactivity was quantifiable from the first sampling point ( 1 hour) in all subjects. Maximum total radioactivity concentrations occurred between 8 and 24 hours and then declined in a multiphasic manner (Fig. 3; Table 1). The median time to maximum concentration $\left(\mathrm{T}_{\max }\right)$ was 10 hours (range: 8-24 hours). Plasma total radioactivity levels remained quantifiable up to the last quantifiable time point ( 168 hours postdose) in all subjects. Terminal slopes were determined for all six subjects with a mean $t_{1 / 2}$ of $\sim 99$ hours (Table 1). Whole-blood and plasma concentrations of total radioactivity were quantified at 1, 6, 24, and 48 hours postdose in all subjects, with mean whole-blood-to-plasma concentration ratios (CV\%) of $1.04(17.6 \%), 1.21(26.8 \%), 0.89$ (24.5\%), and $0.71(13.3 \%)$, respectively.

Ozanimod was quantifiable from the first sampling point (1 hour) in all subjects (Fig. 3). Maximum plasma concentrations occurred between 6 and 12 hours and then declined in a monophasic manner (Fig. 3). The intersubject variability (CV\%) values for ozanimod $\mathrm{C}_{\max }$ and AUC were $22.0 \%$ and $28.5 \%$, respectively. The median $\mathrm{T}_{\max }$ was 8 hours (range: 6-12 hours), and the mean $t_{1 / 2}$ was $\sim 21$ hours. Apparent volume of distribution was 5590 liters, and apparent oral clearance was $192 \mathrm{l} / \mathrm{h}$. Ozanimod represented $\sim 5 \%$ and $12 \%$ of circulating radioactivity in terms of $\mathrm{AUC}_{\text {last }}$ and $\mathrm{C}_{\max }$, respectively, indicating that the majority of circulating radioactivity was attributable to metabolites.

The plasma concentration-time profile of metabolite CC112273 paralleled that of the total radioactivity plasma concentration-time profile. CC112273 exhibited different PK properties compared with

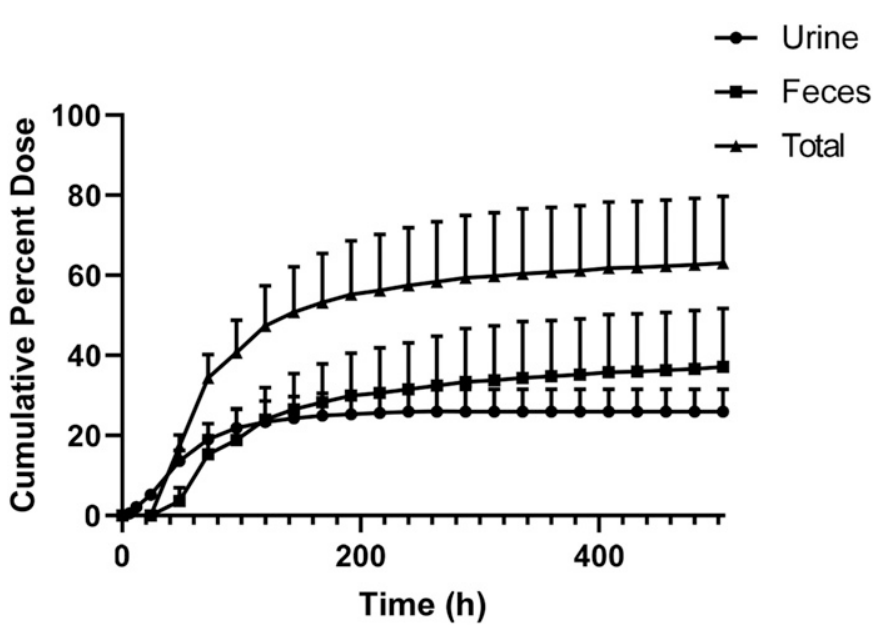

Fig. 2. Mean (+ S.D.) cumulative recovery of total radioactivity (\%CumAe) in urine, feces, and total (urine and feces combined) after a single oral dose of $1 \mathrm{mg}$ $\left[{ }^{14} \mathrm{C}\right]$ ozanimod $\mathrm{HCl}$. 


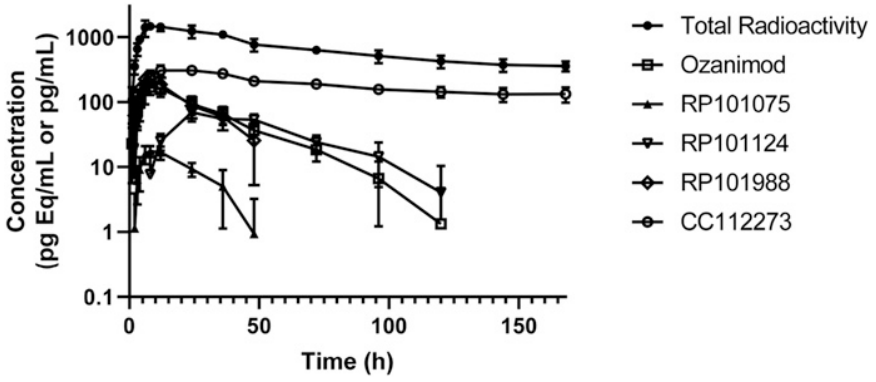

Fig. 3. Mean ( \pm S.D.) plasma concentrations of total radioactivity, ozanimod, and select metabolites after a single oral dose of $1 \mathrm{mg}\left[{ }^{14} \mathrm{C}\right]$ ozanimod $\mathrm{HCl}$.

the parent ozanimod. The median $\mathrm{T}_{\max }$ was 18 hours, and the mean $t_{1 / 2}$ was 195 hours. The metabolites RP101988 and RP101075 showed similar PK properties as the parent ozanimod, with similar $\mathrm{T}_{\max }$ and $t_{1 / 2}$. The metabolite RP101124 showed a delayed $\mathrm{T}_{\max }$ (median 24 hours) and slightly longer $t_{1 / 2}(\sim 28$ hours $)$ compared with ozanimod.

\section{Metabolite Characterization and Metabolic Profiles in Plasma, Urine, and Feces}

The relative amounts of metabolites detected in plasma and excreta are summarized in Table 2. Representative radiochromatograms in excreta and plasma are presented in Figs. 4 and 5, respectively. The proposed mass spectrometry fragmentation pattern for ozanimod is shown in Supplemental Fig. 1, and Table 2 lists the characteristic fragmentation for ozanimod and its metabolites. The structural analysis of metabolites was performed by LC-MS or LC-MS/MS analysis, and the retention times and product ion spectra from metabolites from plasma or excreta were compared with retention time and product spectra of authentic reference standards. For most of the metabolites (with RP or CC number), authentic reference standards were available, and the product spectra matched with the profiled metabolites. The fragmentation data corresponding to each metabolite are shown in Table 2, and fragmentation figures for select prominent metabolites were shown in Supplemental Fig. 2 through Fig. 5.

The abundance of human metabolites of ozanimod that was found in plasma, feces, and urine was presented as the percentage of total radioactivity AUC for circulating metabolites and as the percentage of dose for feces and urine in Table 2. The proposed metabolic pathway of ozanimod in humans is presented in Fig. 6. After oral administration of a single dose of $\left[{ }^{14} \mathrm{C}\right]$ ozanimod, the major circulating components in plasma were $\mathrm{CC} 112273$ and RP101124, with $\sim 33 \%$ and $15 \%$, respectively, of AUC for ${ }^{14} \mathrm{C}$-related drug materials. Ozanimod and the remainder of metabolites are each presented at less than 7\% of AUC for ${ }^{14} \mathrm{C}$-related drug materials after a single dose.
The predominant component recovered in the urine was RP112402, and the predominant components recovered in the feces are RP112533 and RP112480. Ozanimod, CC112273, and RP101075 concentrations in urine were negligible (i.e., below threshold for identification), and RP101988 is the only intact oxadiazole recovered in urine with approximately $4 \%$ of the dose, indicating that renal clearance is not an important excretion pathway for ozanimod or its active metabolites. Approximately $83 \%$ of the recovered radioactive dose was represented by compounds formed as a result of oxadiazole ring reduction and/or scission by gut microflora.

\section{In Vitro Metabolism Experiments}

Characterization of Primary Metabolites. In vitro experiments were conducted to characterize the formation of ozanimod metabolites and the enzymes involved in the metabolism. Ozanimod was stable in human liver microsomes and human hepatocytes, with less than $16 \%$ metabolized over 2 hours. The primary biotransformation of ozanimod occurs via two distinct pathways in vitro: oxidation of primary alcoholic group to corresponding carboxylate metabolite RP101988 and oxidative dealkylation of hydroxyethylamine to form the indanamine metabolite RP101075 (Fig. 6). These primary metabolites underwent further biotransformation resulting in multiple secondary and tertiary metabolites. Phenotyping studies using recombinant cytochrome P450 enzymes and human liver microsomal incubation with cytochrome P450 isozyme selective inhibitors were conducted to identify the enzymes responsible for the formation of RP101075 as well as RP101988.

Characterization of RP101075. Of the recombinant cytochrome P450 enzymes assessed, recombinant CYP3A4 primarily catalyzed the formation of RP101075 from ozanimod (Supplemental Fig. 6A). In human liver microsomal incubation with cytochrome P450 inhibitors, only ketoconazole affected the formation of RP101075 (Supplemental Fig. 6B), further confirming the contribution of CYP3A4 in its formation. The results therefore suggest that the $N$-dealkylation of ozanimod to form RP101075 is primarily mediated by CYP3A4. Incubations in human liver S9 fortified with acetyl CoA and recombinant enzymes show that RP101442 is formed from RP101075 by $\mathrm{N}$-acetylation, and this is mediated by human $\mathrm{N}$-acetyltransferase (NAT) 2, not by NAT1 enzyme (data not shown). RP101442 can also undergo deacetylation to form RP101075 to a limited extent catalyzed by CYP3A4 enzyme. Although CYP3A4 catalyzed dealkylation of $\mathrm{N}$-acetyl group, hydrolysis of amide bond was also observed in control incubations, suggesting esterase/amidase activity.

As noted earlier, the other primary metabolic transformation of ozanimod was oxidation of the primary alcohol to carboxylic acid (RP101988). In vitro studies were conducted to identify the enzymes responsible for this biotransformation using recombinant human cytochrome P450 and other oxidative enzymes, and assessing the effect

TABLE 1

Mean $(\mathrm{CV} \%)$ plasma pharmacokinetic parameters of radioactivity, ozanimod, and its metabolites after single oral dose of $1 \mathrm{mg}\left[{ }^{14} \mathrm{C}\right]$ ozanimod $\mathrm{HCl}$

\begin{tabular}{|c|c|c|c|c|c|c|}
\hline PK Parameter (Unit) & Radioactivity & Ozanimod & $\mathrm{CC} 112273$ & RP101988 & RP101075 & RP101124 \\
\hline $\mathrm{C}_{\max }(\mathrm{pg} / \mathrm{ml})$ & $1560^{a}(7.3 \%)$ & $185(22.0 \%)$ & $337(10.2 \%)$ & $240(21.9 \%)$ & $18.9(18.1 \%)$ & $69.8(27.4 \%)$ \\
\hline $\mathrm{T}_{\max }(\mathrm{h})^{b}$ & $10.00(8.00-24.00)$ & $8.00(6.00-12.18)$ & $18(12-36)$ & $8.00(6.00-12.18)$ & $8.00(6.00-12.18)$ & $24.00(24.00-36.02)$ \\
\hline $\mathrm{AUC}_{\text {last }}(\mathrm{pg} \cdot \mathrm{h} / \mathrm{ml})$ & $116000^{c}(11.8 \%)$ & $5400(29.8 \%)$ & $31000(8.20 \%)$ & $4810(30.2 \%)$ & $376(30.3 \%)$ & $3480(27.3 \%)$ \\
\hline $\operatorname{AUC}_{\infty}(\mathrm{pg} \cdot \mathrm{h} / \mathrm{ml})$ & NR & $5690(28.5 \%)$ & NR & $5630(28.5 \%)$ & NR & $4220(21.5 \%), n=5$ \\
\hline$t_{1 / 2}(\mathrm{~h})$ & $98.61(13.3 \%)$ & $20.78(15.4 \%)$ & $195(52.8 \%), n=4$ & $15.29(17.4 \%)$ & $22.89(48.2 \%) n=4$ & $27.79(18.6 \%) n=5$ \\
\hline $\mathrm{CL} / \mathrm{F}(1 / \mathrm{h})$ & NA & $192(36.9 \%)$ & NA & NA & NA & NA \\
\hline $\mathrm{V}_{\mathrm{z}} / \mathrm{F}(\mathrm{l})$ & NA & $5590(26.6 \%)$ & NA & NA & NA & NA \\
\hline
\end{tabular}

$\mathrm{CL} / \mathrm{F}$, apparent total clearance; NA, not applicable; NR, not reportable; $\mathrm{V}_{\mathrm{z}} / \mathrm{F}$, apparent volume of distribution.

${ }^{a}$ Picogram equivalent per milliliter.

${ }^{b}$ Median (range).

${ }^{c}$ Picogram equivalent hour per milliliter. 
TABLE 2

Metabolite profiles after a single oral dose of $1 \mathrm{mg}\left[{ }^{14} \mathrm{C}\right]$ ozanimod $\mathrm{HCl}$ in healthy subjects

\begin{tabular}{|c|c|c|c|c|c|}
\hline Component & {$[\mathrm{M}+\mathrm{H}]^{+}$} & MS Fragment Ions & $\begin{array}{c}\text { Plasma (\% of } \mathrm{AUC}_{0-96 \mathrm{~h}} \text { for }\left[{ }^{14} \mathrm{C}\right] \text { Related } \\
\text { Drug Materials) }\end{array}$ & Feces (\% of Dose) & Urine (\% of Dose) \\
\hline Ozanimod & 405 & $344,302,188,157,146$ & 6.70 & - & - \\
\hline CC112273 & 360 & $318,188,146,56$ & 33.2 & - & - \\
\hline RP101124 & $204 *$ & 160,133 & 14.5 & 5.53 & 0.73 \\
\hline RP101988 & 419 & $344,302,188,146$ & 6.98 & - & 3.54 \\
\hline RP101075 & 361 & $344,302,188,146$ & 5.40 & - & - \\
\hline RP101442 & 403 & $344,302,188,157,146,60$ & Trace & - & - \\
\hline CC1084037 & 362 & $344,32,188,146$ & 5.48 & - & - \\
\hline RP112289 & 375 & $333,171,146$ & 4.69 & - & - \\
\hline RP112509 & 376 & $358,316,146$ & 3.92 & - & - \\
\hline RP112402 & $380^{*}$ & $204,175,117,113$ & - & - & 15.70 \\
\hline RP112533 & $162 *$ & 118,90 & - & 7.67 & - \\
\hline RP112480 & 422 & $347,188,146,115$ & - & 12.23 & - \\
\hline RP112374 & 408 & $347,188,146,115$ & - & 5.30 & - \\
\hline RP112479 & 421 & $346,188,146$ & - & 2.54 & - \\
\hline M339 & $338 *$ & $162,118,90$ & - & - & 2.58 \\
\hline Total & & & 81.0 & 30.7 & 22.6 \\
\hline
\end{tabular}

—, not present (below the threshold for metabolite identification in specified matrix); $\mathrm{AUC}_{0-96 \mathrm{~h}}$, area under the plasma concentration-time curve from time $0-96 \mathrm{~h}$ postdose, * $=[\mathrm{M}-\mathrm{H}]^{-}$negative mode.

of chemical inhibitors of cytochrome P450 isozymes as well as non-cytochrome P450 enzymes disulfiram [aldehyde dehydrogenase $(\mathrm{ALDH})]$ and 4-methylpyrazole $(\mathrm{ADH})$ on the formation of RP101988 or RP101075 from ozanimod by human liver microsomes in the presence of NAD+ and NADPH was tested. In vitro incubation with recombinant cytochrome P450 enzymes showed that RP101988 is not formed by any of the 14 cytochrome P450 enzymes. Although ketoconazole showed some inhibition, the role of CYP3A4 involvement was not supported by another CYP3A4 specific inhibitor or lack of formation in incubations with recombinant cytochrome 3A4 (rCYP3A4). Studies with human liver microsomes and semicarbazide trapping suggests the formation of RP101988 proceeds via an aldehyde intermediate. In addition, the formation of RP101988 increased 2-fold at $\mathrm{pH} 8.5$ compared with pH 7.5 or with NAD+ compared with NADPH, suggesting the involvement of dehydrogenases. Furthermore, an ALDH inhibitor,

A

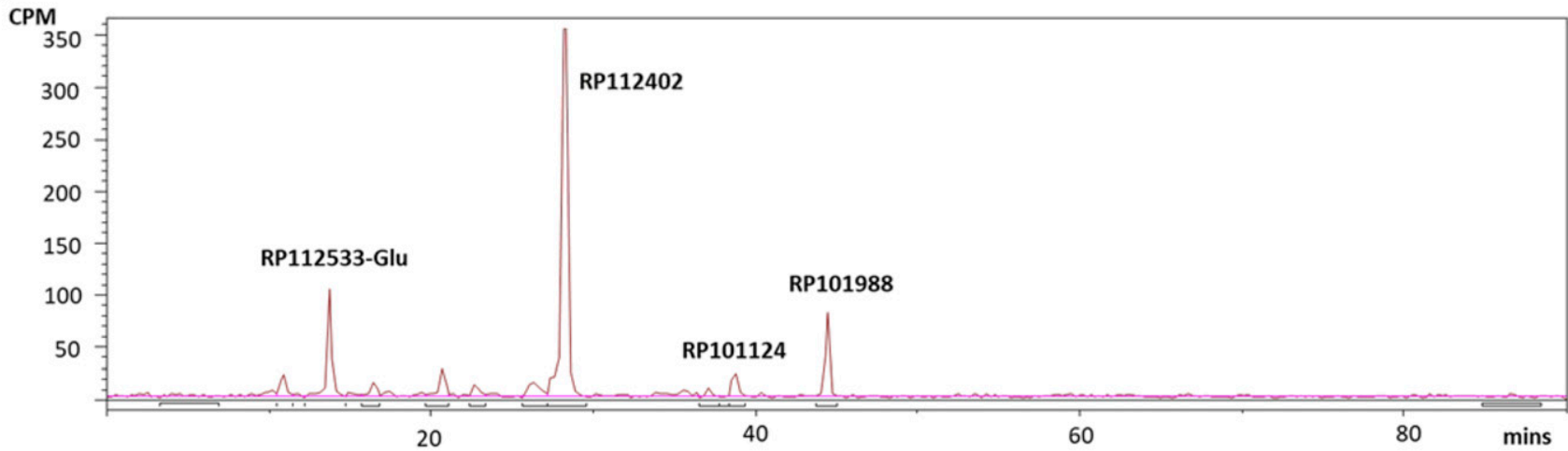

B

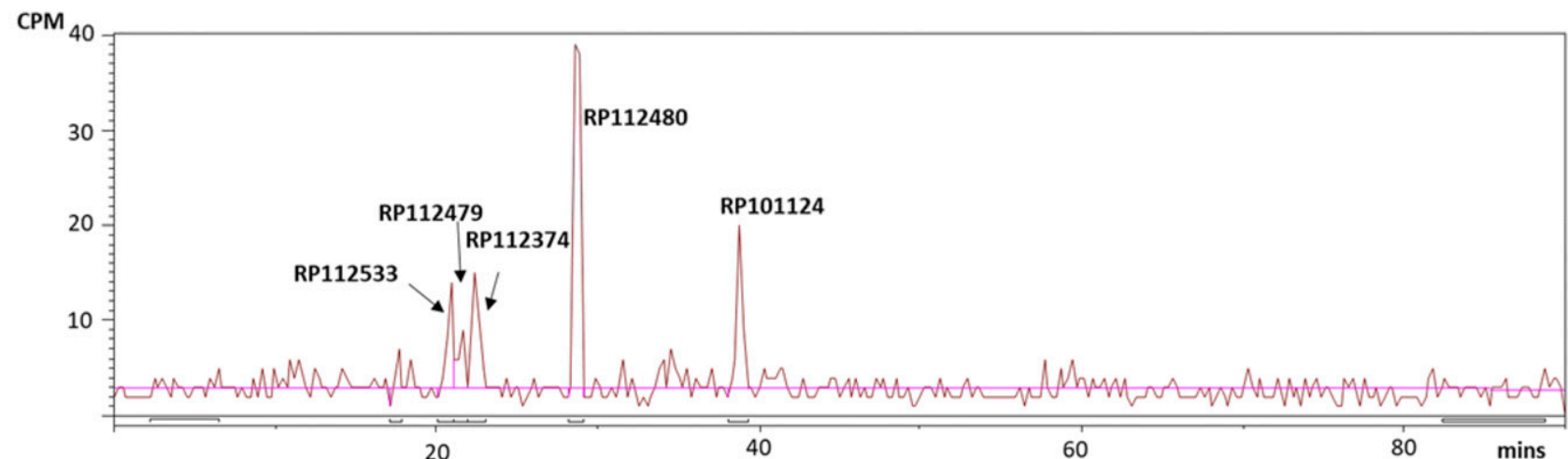

Fig. 4. Representative HPLC radiochromatograms of metabolic profile [counts per minute (CPM) vs Time] in pooled urine (A) and feces (B). 


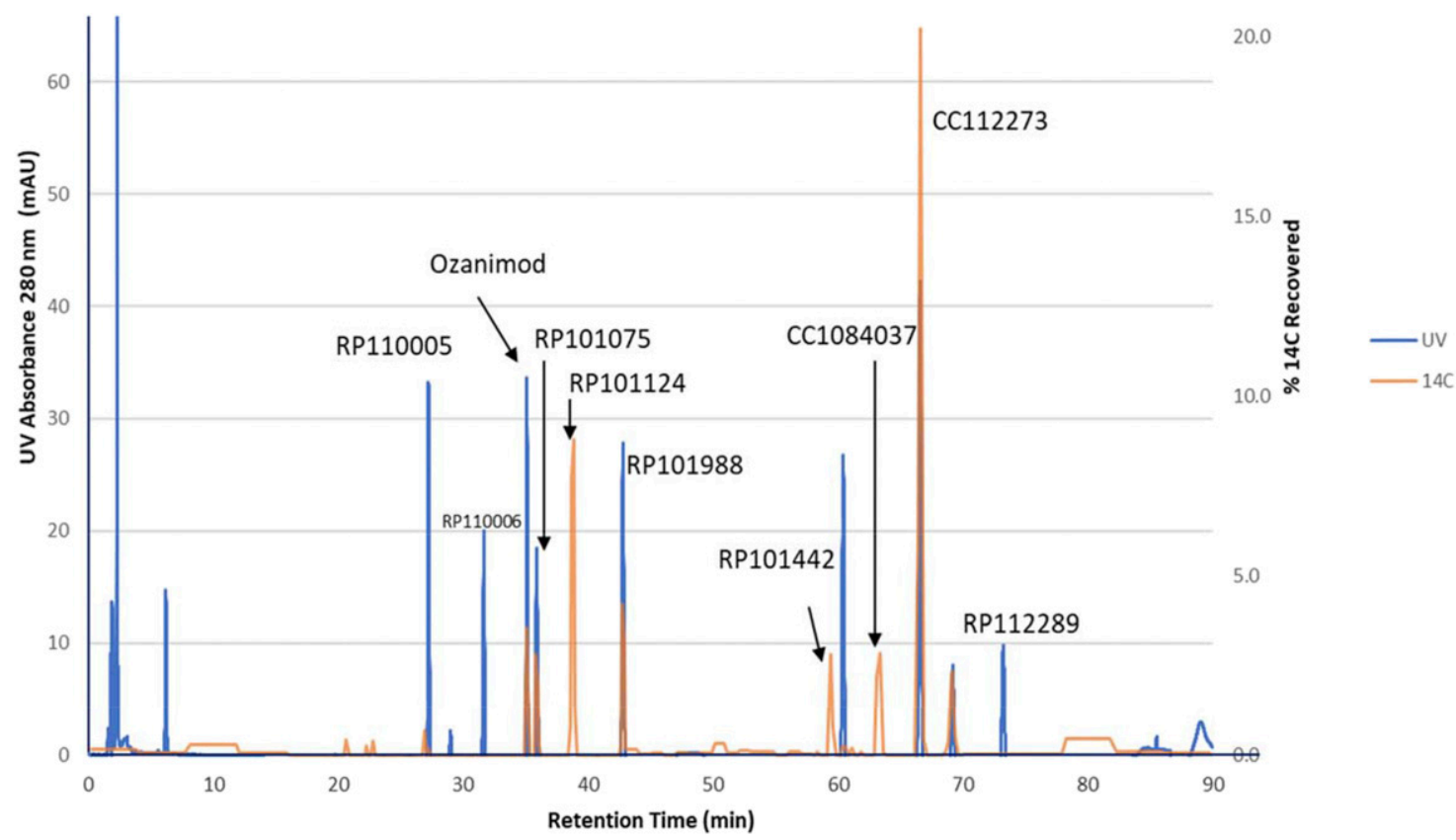

Fig. 5. Representative HPLC radiochromatogram of metabolic profile in pooled human plasma.

disulfiram, and ADH inhibitor, 4-methylpyrazole, inhibited the formation of RP101988 (https://www.accessdata.fda.gov/ drugsatfda_docs/nda/2020/209899Orig1s000PharmR.pdf). Based on the collective data, the formation of RP101988 is mediated by non-cytochrome P450 enzymes ADH and ALDH working in tandem to convert alcohol functional group to carboxylic acid.

To identify the enzymes involved in formation of major human metabolite CC112273, incubations of RP101075 were performed in HLM in the presence and absence of cytochrome P450 chemical inhibitors, including the nonspecific cytochrome P450 inhibitor 1-aminobenzotriazole. The amount of RP101075 remaining after 60-minute incubations in the presence and absence of chemical inhibitors ranged from $80 \%$ to $99 \%$. However, little to no inhibition of CC112273 formation was observed in the presence of directacting or metabolism-dependent cytochrome P450 inhibitors (Fig. 7A). These results indicated that cytochrome P450 enzymes did not play a role in formation of CC112273 from RP101075 or ozanimod. However, the formation of RP112289 was inhibited by mechanism-based CYP3A4 inhibitor troleandomycin by $75 \%$ inhibition (Fig. 7B). The recombinant enzyme data showed that CYP3A4 predominantly catalyzed formation of RP112289 (Fig. 7C). From these experiments, it was clear that metabolite RP112289 is formed by

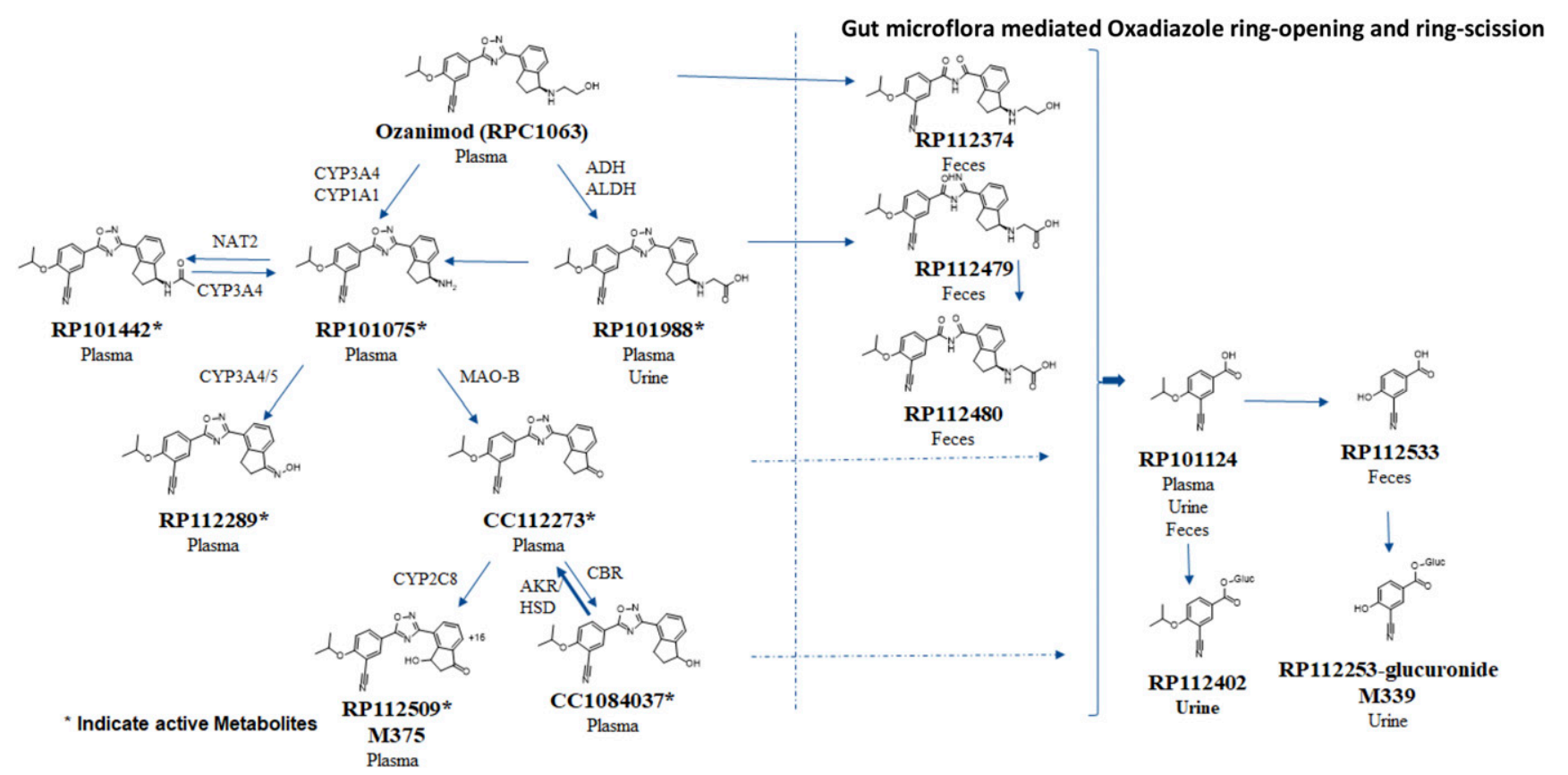

Fig. 6. Proposed metabolic pathways of ozanimod in humans. 
A

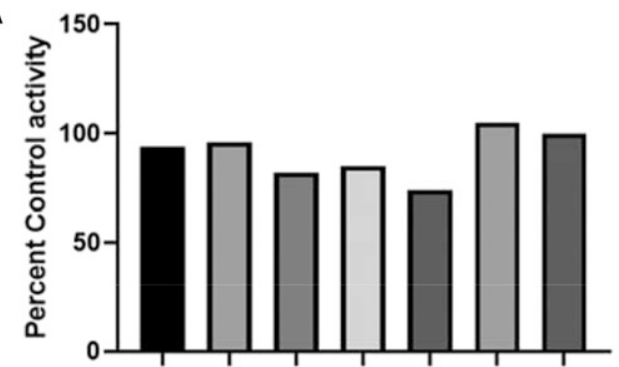

$0 \perp$

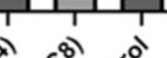

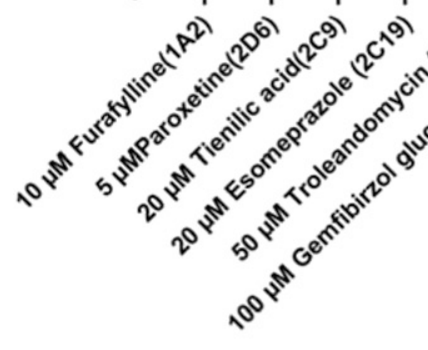

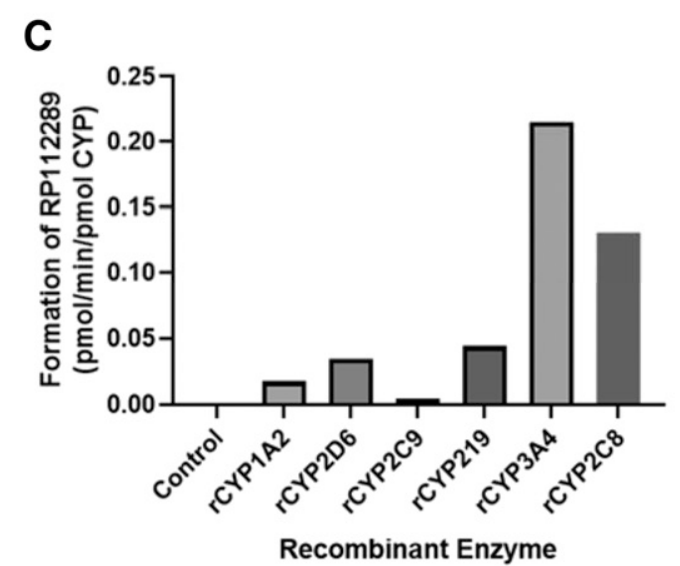

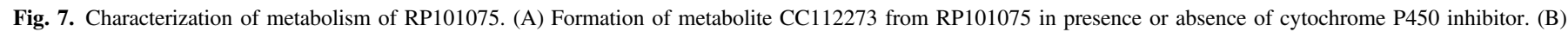
Formation of RP112289 from RP101075 in presence of cytochrome P450 selective inhibitors. (C) Formation of RP112289 from RP101075 by recombinant.

CYP3A4/A5, whereas CC112273 is formed by non-cytochrome P450 enzymes.

CC112273 was formed when ozanimod or RP101075 was incubated with or without NADPH and human liver microsomes, S9, mitochondria, or cytosol (Fig. 8A). As shown with selective chemical inhibitor, cytochrome $\mathrm{P} 450$ enzymes are unlikely to be involved in the formation of CC112273. Incubations of RP101075 with monoamine oxidases (MAO-A and MAO-B) showed that MAO-B is capable of forming CC112273, whereas MAO-A did not catalyze the formation of CC112273 (Fig. 8B). The selective inhibitor of MAO-A, chlorgyline, did not show any inhibition of formation of $\mathrm{CC} 112273$, whereas the MAO-B inhibitor, deprenyl, completely inhibited the formation of CC112273 from RP101075. Based on the collective data, it was concluded that MAO-B is the enzyme responsible for the formation of CC112273 from RP101075 and that CC112273 is not formed directly from ozanimod but requires prior formation of RP101075 (Fig. 8C).

Two downstream metabolites of CC112273, CC1084037 and RP112509 (also known as M375), were formed via reduction and oxidation, respectively. To characterize these metabolites and elucidate the enzymes responsible for the formation or metabolism of these metabolites, incubations of $\mathrm{CC} 112273$ with various matrices were conducted. The formation of $\mathrm{CC} 1084037$ required the presence of NADPH, and both human liver microsomes and liver S9 were capable of forming this metabolite. The formation of CC1084037 from CC112273 was not inhibited by known cytochrome P450 inhibitors, such as furafylline (CYP1A2), quercetin (CYP2C8, CBR1), sulfaphenazole (CYP2C9), quinidine (CYP2D6), ketoconazole (CYP3A4 plus
B

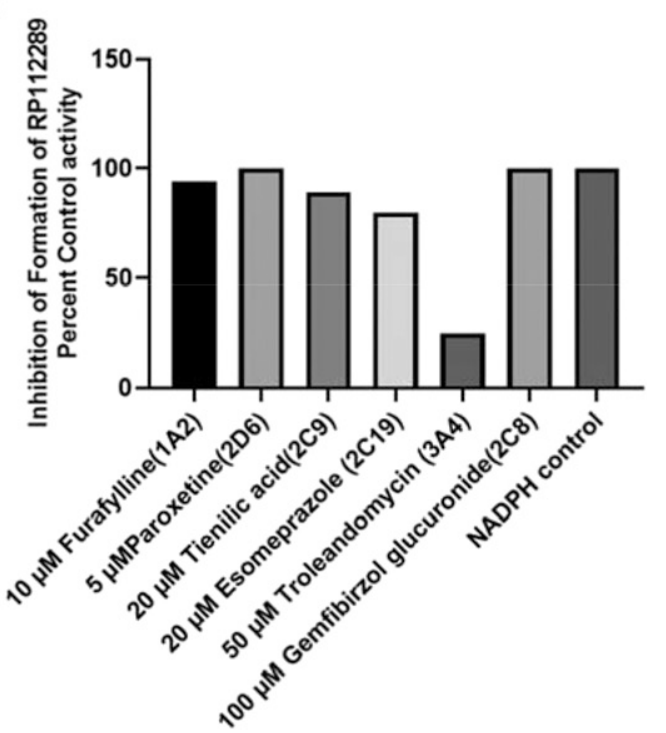
showed that $\mathrm{CC} 1084037$ is a direct metabolite of CC112273, with carbonyl reductases as the catalytic enzymes involved. Since CC1084037 was a downstream metabolite of $\mathrm{CC} 112273$, its metabolism was investigated using human hepatocytes, liver cytosol, and HLM. CC1084037 is rapidly oxidized to $\mathrm{CC} 112273$ in human hepatocytes and in human cytosol or microsomes in the presence of $\mathrm{NADP}^{+}$or $\mathrm{NAD}^{+}$. The relative rates of formation of CC112273 from CC1084037 and CC1084037 from CC112273 indicate that the oxidative pathway predominates over the reductive pathway. Identification of enzymes responsible for the metabolism of CC1084037 using recombinant enzymes and selective inhibitors indicated that the conversion of CC1084037 to CC112273 was mediated by multiple enzymes, including AKRs, namely AKR1C1 and AKR1C2, and HSDs, namely $3 \beta$-HSD and $11 \beta$-HSD (Fig. 9, B and C). There were no direct oxidative or conjugated metabolites of CC1084037 found in vitro in human hepatocytes, other than the conversion to CC112273 and its subsequent metabolism. This indicates that CC1084037 and CC112273 are interconvertible, with the predominant circulating species being CC112273. The elucidation of the 
A

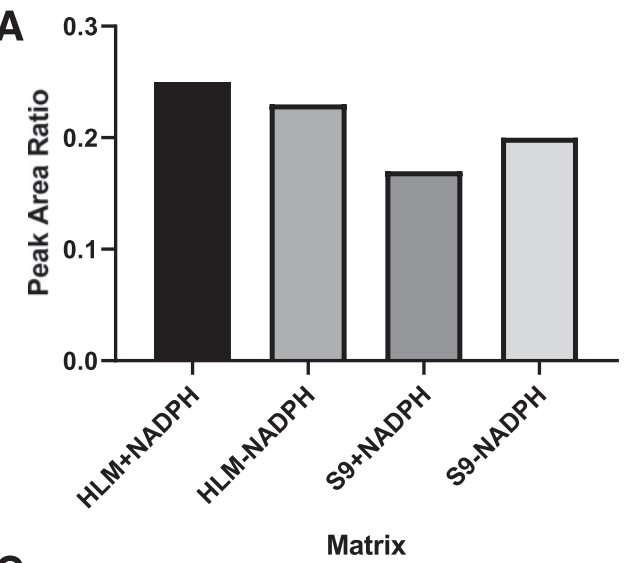

C

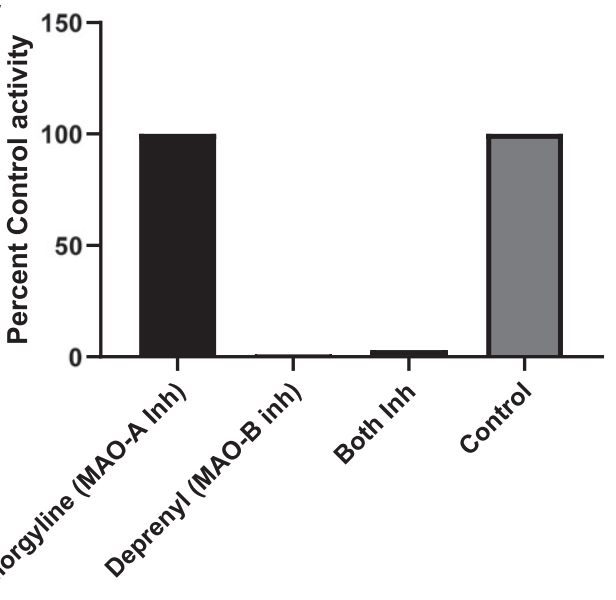

B

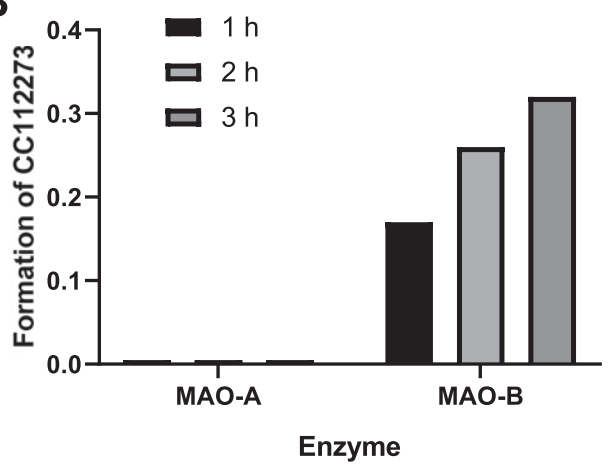

oxidoreduction mechanism of CC112273 and CC1084037 and their interconversion kinetics provide an explanation for the predominance of CC112273 in human plasma.

A second downstream metabolite of CC112273, RP112509, resulted from mono-oxidation of the indane ring. The exact position of oxidation was determined using human liver subcellular fractions, mass spectral fragmentation, and comparison of the retention time and mass spectral fragmentation with the authentic reference standard. The formation of RP112509 was inhibited by the CYP2C8 inhibitor quercetin. Based on these phenotyping results, a subsequent clinical drug-drug interaction study confirmed the finding that CYP2C8 is responsible for clearance of CC112273 via RP112509.

Mass balance and metabolite profiling study in rat as well as human mass balance studies identified RP101124 as a major inactive metabolite in circulation. Based on the structure, it is evident that RP101124 formed as a result of scission of the oxadiazole ring system (Fig. 6). In an in vitro study, freshly collected feces on day 5 from rats after 5 days of treatment with antibiotics or vehicle (or untreated) were cultured in thioglycollate media (T9997; TekNova) at $37^{\circ} \mathrm{C}$ in an Oxoid Anaerobic Pouch System and incubated with ozanimod or RP101988, and samples were analyzed for their metabolites. The methylene blue assay was used to measure the presence of bacteria in anaerobic cultures. After a 24-hour incubation of ozanimod in rat fecal cultures, $10 \%$ of the original ozanimod concentration was converted to RP101124 in cultures from vehicletreated rats, but no conversion was observed in cultures from rats dosed with antibiotics for 5 days (Fig. 10A). In addition, in vitro study with either ozanimod or metabolite RP101988 for 6 hours under anaerobic conditions showed that the formation of metabolite RP101124 is mediated by gut microbial metabolism and that the absence of bacteria or anaerobic conditions precludes the formation of RP101124 (Fig. 10, B and C). These experiments indicate that RP101124 is not formed systemically but rather in the gut under anaerobic conditions and absorbed into systemic circulation.

To investigate the potential loss of ${ }^{14} \mathrm{C}$ label, RP112533 (the fecal metabolite of ozanimod) was incubated with human fecal homogenates for 96 hours to quantitate the loss of radioactivity as well as formation of the resulting metabolite 2-hydroxy benzoic acid (Fig. 11). As shown in the graph, fecal incubations under anaerobic conditions resulted in loss of radioactivity, and in the presence of antibiotics, this loss could be prevented (Fig. 11A). In addition, corresponding product 2-hydroxy benzonitrile formed only in incubations without antibiotic and not in control incubations or with antibiotics, showing that anaerobic bacterial metabolism leads to loss of $\mathrm{CO}_{2}$ (Fig. 11B). These results suggested the potential for decarboxylation of RP112533 to occur in vivo, resulting in loss of radiolabel as ${ }^{14} \mathrm{CO}_{2}$ in expired air.

S1P Receptor Profile and In Vitro Activity. Ozanimod is a selective modulator for human $\mathrm{S}_{1} \mathrm{P}_{1}$ and $\mathrm{S}_{1} \mathrm{P}_{5}$ and induced robust $\left[{ }^{35} \mathrm{~S}\right] \mathrm{GTP} \gamma \mathrm{S}$ binding in membranes prepared from $\mathrm{CHO}$ cells expressing the $\mathrm{S}_{1} \mathrm{P}_{1}$ and $\mathrm{S}_{1} \mathrm{P}_{5}$ human receptor subtypes. The activity of ozanimod at human $\mathrm{S}_{1} \mathrm{P}_{2}$, human $\mathrm{S}_{1} \mathrm{P}_{3}$, or human $\mathrm{S}_{1} \mathrm{P}_{4}$ was weak, not achieving relative intrinsic activity above $50 \%$ of that of the endogenous ligand, S1P, and with potencies that would not enable target engagement at the observed clinical exposures. This profile was also true for the active metabolites of ozanimod, CC112273, CC1084037, RP101075, RP101988, RP101442, 
A

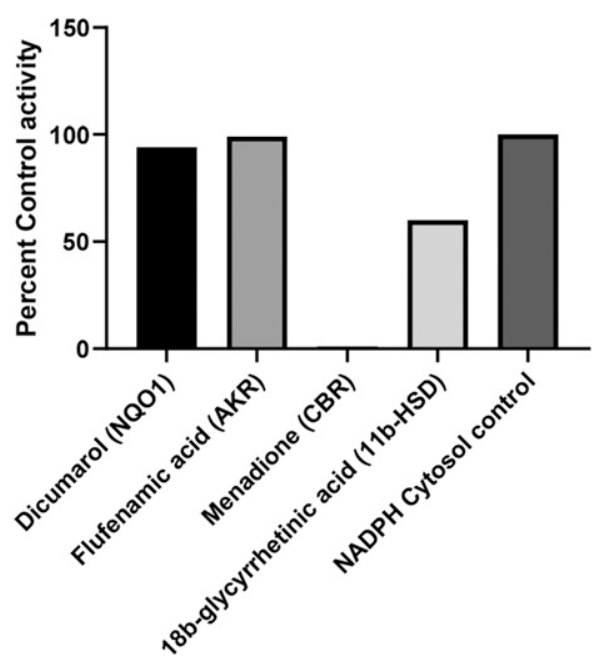

C
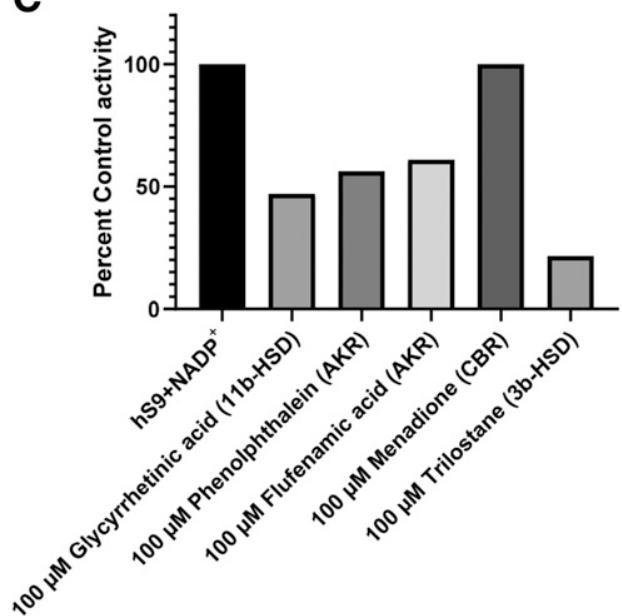

B

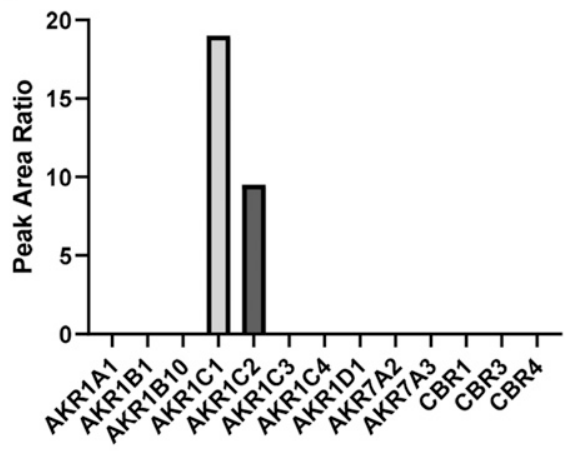

Fig. 9. Characterization of formation and metabolism of CC1084037. (A) Inhibition of formation of CC1084037 when incubated with CC112273 in human liver cytosol in presence of NAPDH and selective inhibitors of $\mathrm{NAD}(\mathrm{P}) \mathrm{H}$ quinone dehydrogenase 1 (NQO1), aldoketo reductases (AKR), carbonyl reductases (CBR), 11 betahydroxysteriodal dehydrogenases (11b-HSD). (B) Formation of CC112273 when incubated with CC1084037 and NADP ${ }^{+}$with recombinant enzymes. (C) Inhibition of formation of CC112273 with selective inhibitors of AKR, CBR, and HSD enzymes in human liver S9 (hS9) and NADP ${ }^{+}$.

RP112289, and RP112509. RP101124 was determined to be an inactive metabolite across all five human S1P receptor subtypes since it did not elicit measurable $\left[{ }^{35} \mathrm{~S}\right] \mathrm{GTP} \gamma \mathrm{S}$ binding across $\mathrm{S}_{1} \mathrm{P}_{1}-\mathrm{S}_{\mathrm{P}}$. As such, the active metabolites of ozanimod all demonstrate a similar activity profile to the parent compound in that they are potent robust modulators for $\mathrm{S}_{1} \mathrm{P}_{1}$ and $\mathrm{S}_{1} \mathrm{P}_{5}$ with demonstrated selectivity over $\mathrm{S}_{1} \mathrm{P}_{2}, \mathrm{~S}_{1} \mathrm{P}_{3}$, and $\mathrm{S}_{1} \mathrm{P}_{4}$ (Table 3).

\section{Discussion}

After a single oral dose of $1 \mathrm{mg}\left[{ }^{14} \mathrm{C}\right]$ ozanimod $\mathrm{HCl}$, ozanimod was readily absorbed, with peak plasma concentrations reaching between 8 and 24 hours, and then declined in a multiphasic manner, consistent with extravascular administration of drug. Whole-blood-to-plasma concentration of total radioactivity ratios ranged from 0.71 to 1.21 , suggesting no preferential binding to blood cells either by ozanimod or its metabolites. The plasma PK parameters for ozanimod were consistent with what was observed in other studies. The $t_{1 / 2}$ for total radioactivity ranged from 84 hours to 117 hours, with a mean $t_{1 / 2}$ of 99 hours. In contrast, ozanimod exhibited a mean $t_{1 / 2}$ of 21 hours, indicating that the metabolites contributed to the long terminal $t_{1 / 2}$ of total radioactivity. Indeed, the mean $t_{1 / 2}$ of metabolite CC112273 was 195 hours. The parent drug, ozanimod, represented approximately $6.7 \%$ of circulating radioactivity in terms of $\mathrm{AUC}_{\text {last }}$, whereas the combined ozanimod, RP101988, RP101075, and RP101124 AUC last levels accounted for approximately $33.6 \%$ of the circulating total radioactivity. CC112273 was the most predominant metabolite after a single oral dose of $\left[{ }^{14} \mathrm{C}\right]$ ozanimod and accounted for $33 \%$ of the circulating radioactivity exposure and exhibited longer $t_{1 / 2}$ than ozanimod or metabolites RP101988, RP101075, and RP101124.

Ozanimod is an interesting case study that highlights the importance of doing the radiolabeled human AME studies at the right time during the drug development. Prior to conducting the human radiolabeled study, metabolites RP101988, RP101075, RP101124, and RP101442 were identified by in vitro methods and monitored in preclinical and clinical studies as they were either active and/or present at similar or higher levels than ozanimod (https://www. accessdata.fda.gov/drugsatfda_docs/nda/2020/

209899Orig1s000PharmR.pdf). Although a rodent radiolabeled study was conducted, the complex metabolic pathway of ozanimod resulted in quantitative differences in circulating exposures of metabolites due to differences in clearance and half-life in rat and humans despite qualitatively similar metabolic profiles. As a result of these quantitative differences, CC112273 was present at low levels in rat because of lower extent of formation and higher clearance, and the 
A
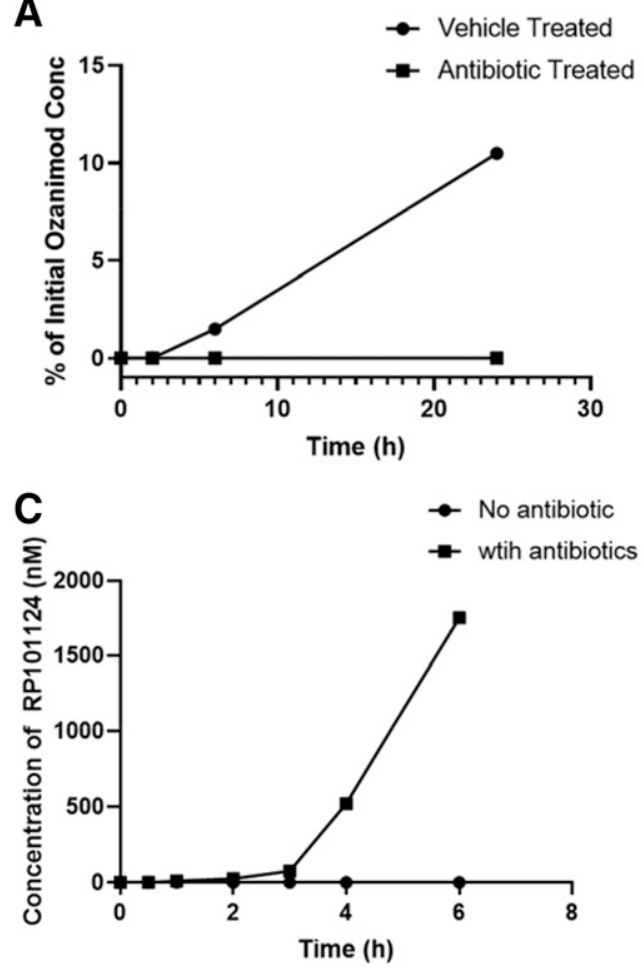

B

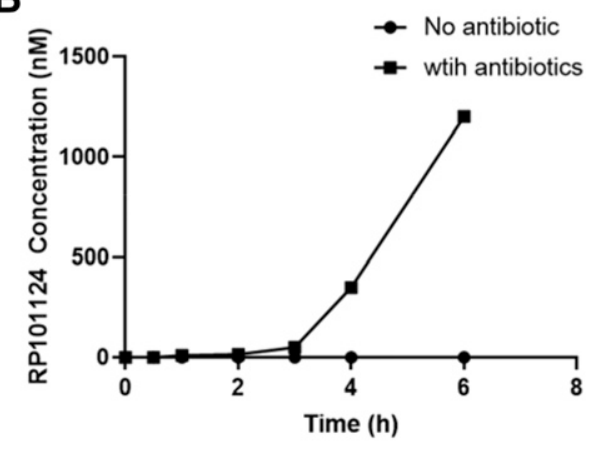

radiolabeling study failed to identify this metabolite prior to human AME results (https://www.accessdata.fda.gov/drugsatfda_docs/nda/ 2020/209899Orig1s000PharmR.pdf). The major human disproportionate metabolite CC112273 was not identified until late in the development when the radiolabeled study described here was conducted. After identification of CC112273 as a major circulating, disproportionate, and active metabolite with long half-life, steadystate exposures were determined in multiple-dose studies in patients with relapsing multiple sclerosis, which showed that metabolite CC112273 accumulated approximately 11- to 13-fold upon repeat dosing, whereas the parent exhibited 2-fold accumulation, consistent with their $t_{1 / 2}$ (Tran et al., 2017; Kuan et al., 2019). The identification of disproportionate metabolites late in the development presented challenges for the metabolites in safety testing assessment and pharmacokinetic-pharmacodynamic and exposure-response assessment in clinical pharmacology studies. To demonstrate exposure coverage in chronic, reproductive, and carcinogenicity toxicology studies, bridging repeat-dose good laboratory practice PK studies in preclinical species (rat, mouse, rabbit, and monkey) were conducted with ozanimod, and exposures of disproportionate metabolites were generated to calculate safety multiples. In addition, exposures of major active metabolites were assessed in the clinical pharmacology studies to build the exposure-response and drug-drug interaction characterization. Identification of disproportionate metabolites late in development presented formidable challenges and delays highlighting the criticality of human ADME data and the need for conducting these studies at the right time during development.

After administration of a single $1-\mathrm{mg}$ oral dose of $\left[{ }^{14} \mathrm{C}\right]$ ozanimod $\mathrm{HCl}$, an average of $63 \%$ (range from $41 \%$ to $85 \%$ ) of the radioactivity administered was recovered in urine and feces over the 504-hour sampling period. Approximately $26 \%$ (range from $19 \%$ to $32 \%$ ) of the radioactive dose was recovered in the urine samples collected up to 10 days postdose, with a further $37 \%$ (range from $21 \%$ to $58 \%$ ) recovered in the feces samples. The total recovery of radioactivity was low $(63 \%)$. The percent of ozanimod in urine was low $(\sim 0.2 \%)$, and computed renal clearance based on this recovery was $5.73 \mathrm{ml} / \mathrm{min}$, indicating that urinary clearance is not a major route of elimination for intact ozanimod. One of the circulating metabolites, RP101988, representing $2.59 \%$ of the dose excreted in urine and the renal clearance of this metabolite indicates that urinary excretion is a notable pathway, and this is consistent with the carboxylic acid functional group, charge, and polarity of this molecule. The predominant component of urinary radioactivity was RP112402, accounting for $15.7 \%$ of the dose excreted in urine. Metabolite RP112402 is a glucuronide metabolite of RP101124, which is formed as a result of oxadiazole ring scission in the gut by microbial flora. The metabolite RP101124 is subsequently absorbed and glucuronidated in the liver to form RP112402 and eliminated predominantly via urinary excretion. Reductive cleavage of $\mathrm{N}-\mathrm{O}$ bond in isoxazole and oxadiazole ring systems is well documented in the literature, and a common pathway for oxadiazole is reductive cleavage followed by hydrolysis resulting in ring scission (Yabuki et al., 1993; Dalvie et al., 2002; Zhang et al., 2008). The fecal radioactivity mainly consisted of ring scission metabolites, formed via anaerobic microbial biotransformation activity. Since these metabolites potentially originated from unabsorbed ozanimod or any metabolites or parent excreted via the hepatobiliary pathway, estimation of the percent of drug absorbed from this study proved to be challenging.

The mass balance data indicated low recovery of $63 \%$. The low recoveries of radioactivity for drugs with a long $t_{1 / 2}$ are well documented in literature. One of the potential reasons for low recovery is a very long 
A

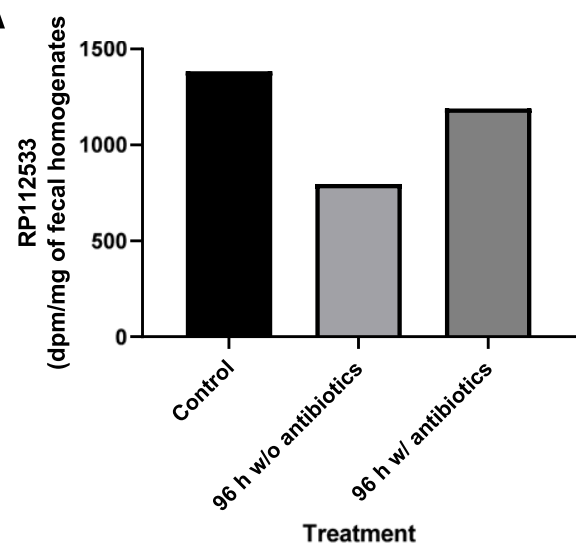

B

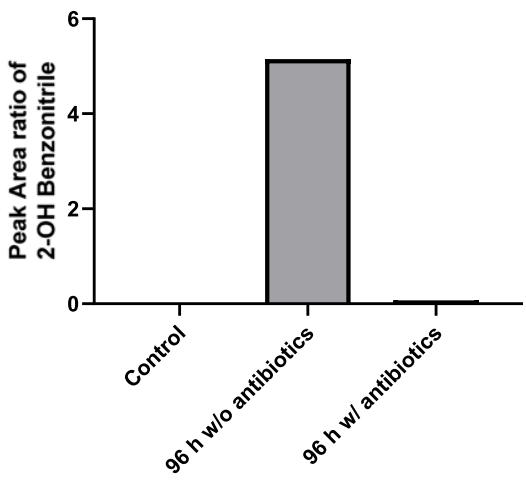

C

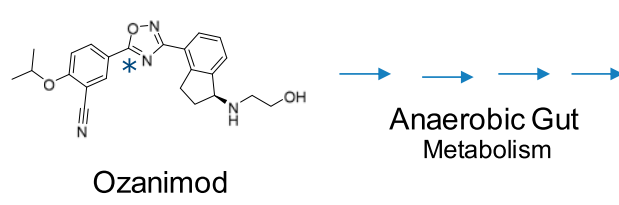

+ Other metabolites

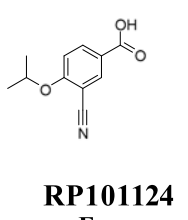

Feces

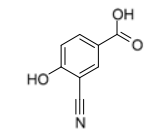

RP112533

Feces

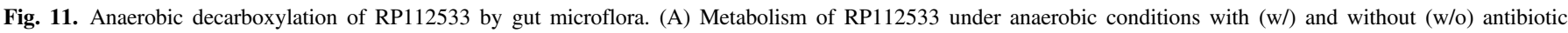

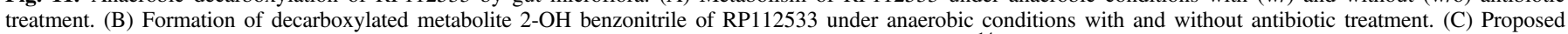
pathway to ultimate fate of ozanimod or its metabolites in gut under anaerobic conditions and release of ${ }^{14} \mathrm{CO}_{2}$.

plasma radioactive $t_{1 / 2}$ leading to dilution of drug-related material in excreta such that radioactivity in samples is below the limit of quantification (Roffey et al., 2007). When the circulating $t_{1 / 2}$ of total radioactivity is greater than 50 hours, the recovery tends to be lower. Of note, the total radioactivity recovered in this study was also similar to that reported for fingolimod, an approved S1P modulator, in which the observed excretion of radioactivity was slow and incomplete (62\%), consistent with the long $t_{1 / 2}$ of total radioactivity (Zollinger et al., 2011). In addition, the role of anaerobic bacterial gut metabolism of ozanimod and its oxadiazole intact metabolites and potential loss of radiolabel via oxadiazole ring scission was investigated. As shown in the metabolism of opicapone, which shares an oxadiazole ring system with ${ }^{14} \mathrm{C}$ label in the same position as in $\left[{ }^{14} \mathrm{C}\right]$ ozanimod, bacterial gut metabolism leads to scission of ring system and finally loss of label as ${ }^{14} \mathrm{CO}_{2}$ (https://www. ema.europa.eu/en/documents/product-information/ongentys-eparproduct-information_en.pdf). It is reported that as much as $10 \%-23 \%$ of radiolabel was accounted for in the expired air (https://www.ema. europa.eu/en/documents/product-information/ongentys-epar-productinformation_en.pdf). Given the precedence, incubations of RP112533 (the fecal metabolite of ozanimod) with human fecal homogenates in vitro for 96 hours under anaerobic conditions resulted in loss of radioactivity, and, in presence of antibiotics, this loss was prevented, implicating the role of anaerobic bacterial metabolism leading to loss of ${ }^{14} \mathrm{CO}_{2}$. In addition, corresponding product 2-hydroxy benzonitrile formed only in incubations without antibiotic and not in control incubations or with antibiotics, showing that anaerobic bacterial metabolism leads to loss of $\mathrm{CO}_{2}$. Taken together, the low recovery of total radioactivity is due to a combination of long $t_{1 / 2}$ of ozanimod metabolites as well as loss of ${ }^{14} \mathrm{C}$ label as carbon dioxide $\left({ }^{14} \mathrm{CO}_{2}\right)$ in the expired air because of anaerobic microbial reductive metabolism of oxadiazole moiety, which was unaccounted for in the mass balance study.
In summary, this human AME helped understand the disposition of ozanimod in humans and enabled identification of previously unknown major metabolite CC112273 and its downstream metabolites. Overall, one active metabolite, $\mathrm{CC} 112273$, and one inactive metabolite, RP101124, were identified to be greater than $10 \%$ of the total radioactivity and warranted further study to characterize the exposure in nonclinical toxicology as well as in clinical studies. Further in vitro metabolism studies identified that CC1084037 is an interconverting downstream metabolite of CC112273 with similar activity profile as parent and CC112273. Although it was only present at approximately $5 \%$ of the total radioactivity in this single-dose study, it was a downstream metabolite of a predominant active and long-lived metabolite CC112273, which accumulated upon multiple dosing. Further analysis of steady-state PK samples showed that CC1084037 exceeded the 10\% threshold and was present at $15 \%$ of the total drug exposure upon repeat dosing of ozanimod. The results indicated that ozanimod undergoes extensive metabolism and is primarily excreted as metabolites in urine and feces. Ozanimod and its active metabolites exhibited similar activity and selectivity for $\mathrm{S}_{1} \mathrm{P}_{1}$ and $\mathrm{S}_{1} \mathrm{P}_{5}$. Both active metabolites $\mathrm{CC} 112273$ and CC1084037 together with ozanimod contribute to the majority of the circulating radioactivity and account for most of the pharmacological activity. Although recovery was low, this was attributed to the long $t_{1 / 2}$ and loss of radiocarbon via carbon dioxide through gut-mediated decarboxylation of labeled metabolite by bacterial microflora under anaerobic conditions.

Despite the low recovery, results from the ADME study provided an understanding of the metabolic profile of ozanimod in humans, thus fulfilling the main objective of the human mass balance study (Roffey et al., 2007). The results from the ADME study helped identify major circulating metabolites CC112273, CC1084037, and RP101124, necessitating further assessment of CC112273 and CC1084037 for adequate exposure in toxicological evaluation as recommended in the current 
regulatory guidelines (ICH, 2010, ICH, 2013, FDA 2016). Ozanimod is extensively metabolized in humans to form a number of circulating active metabolites, including two major active metabolites, CC112273 and CC1084037, and one inactive metabolite, RP101124. Multiple enzyme systems play an important role in the metabolism of ozanimod, and no single enzyme system predominates in the overall metabolism of ozanimod. The oxidative pathway to formation of carboxylate metabolite RP101988 is mediated by aldehyde dehydrogenase and alcohol dehydrogenase, whereas formation of RP101075 by dealkylation is predominantly carried out by cytochrome P450 3A4. RP101075 is $\mathrm{N}$-acetylated by NAT2 to form RP101442 or deaminated by MAO-B to form the major metabolite CC112273. CC112273 is either reduced to form CC1084037 or undergoes CYP2C8-mediated oxidation to form $\mathrm{RP} 101509$. CC1084037 is oxidized rapidly to form CC112273 by AKR $1 \mathrm{C} 1 / 1 \mathrm{C} 2$ and/or $3 \beta$ - and $11 \beta$-HSD. The oxidoreduction interconversion between $\mathrm{CC} 112273$ and $\mathrm{CC} 1084037$ favors $\mathrm{CC} 112273$, and there are no direct metabolites of $\mathrm{CC} 1084037$ other than its metabolism back to CC112273 and subsequent elimination via that pathway. Gut microbial flora play an important role in vivo via anaerobic reductive metabolism of the oxadiazole ring system in the formation of many inactive metabolites, which constitute a predominant portion of the excreted dose via urine and feces.

\section{Acknowledgments}

The authors would like to thank Brahmachary Enugurthi, Maurice Marsini, and Roger Bakale (Process Chemistry, Celgene) for the preparation of authentic standards for metabolites and facilitating synthesis of radiolabeled ozanimod. The authors would also like to thank E. van Duijn and R.A.F. de Ligt at TNO; Debra Beck at ICON (Whitesboro, NY); and Eleanor Barton, Caroline Clegg, Dylan Williams, Marc McCarthy, and Rebecca Shellard at Pharmaron (Nottingham, UK) for their technical assistance.

\section{Authorship Contributions}

Participated in research design: Surapaneni, Yerramilli, Bai, Dalvie, Brooks, Wang, Selkirk, Yan, Zhang, Kumar, Palmisano, Tran.

Conducted experiments: Yerramilli, Bai, Dalvie, Brooks, Wang, Selkirk, Yan, Tran.

Performed data analysis: Surapaneni, Yerramilli, Bai, Dalvie, Brooks, Wang, Selkirk, Yan, Zhang, Tran.

Wrote or contributed to the writing of the manuscript: Surapaneni, Yerramilli, Bai, Dalvie, Brooks, Wang, Selkirk, Yan, Zhang, Hargreaves, Kumar, Palmisano, Tran.

Note Added in Proof: A typo was found in the title of this article in the Fast Forward version published March 4, 2021. The title has now been corrected.

\section{References}

Brinkmann V, Davis MD, Heise CE, Albert R, Cottens S, Hof R, Bruns C, Prieschl E, Baumruker T, Hiestand P, et al. (2002) The immune modulator FTY720 targets sphingosine 1-phosphate receptors. J Biol Chem 277:21453-21457.

Brinkmann V (2009) FTY720 (fingolimod) in Multiple Sclerosis: therapeutic effects in the immune and the central nervous system. Br J Pharmacol 158:1173-1182.

Coppola P, Andersson A, and Cole S (2019) The importance of the human mass balance study in regulatory Submissions. CPT Pharmacometrics Syst Pharmacol 8:792-804.

Dalvie DK, Kalgutkar AS, Khojasteh-Bakht SC, Obach RS, and O’Donnell JP (2002) Biotransformation reactions of five-membered aromatic heterocyclic rings. Chem Res Toxicol 15:269-299.

FDA (2020) Guidance for Industry: Safety Testing of Drug Metabolites, US Department of Health and Human Services FaDA, Center for Drug Evaluation and Research, Silver Spring, MD.

Feagan B G Sandborn WJ, Danese S, Wolf DC, Liu WJ, Hua SY, Minton N, Olson A, D'Haens G. (2020) Ozanimod induction therapy for patients with moderate to severe Crohn's disease: a single-arm, phase 2, prospective observer-blinded endpoint study. Lancet Gastroenterol Hepatol 5 (9):819-828.

ICH (2010) M3(R2) Nonclinical Safety Studies for the Conduct of Human Clinical Trials and Marketing Authorization for Pharmaceuticals, The International Council for Harmonisation of Technical Requirements for Pharmaceuticals for Human Use, ICH, Geneva, Switzerland.

ICH (2013) M3(R2) Nonclinical safety studies for the conduct of human clinical trials and marketing authorization for pharmaceuticals. Questions and Answers(R2), The International Council for Harmonisation of Technical Requirements for Pharmaceuticals for Human Use, ICH, Geneva, Switzerland.

Karuppuchamy T, Behrens EH, González-Cabrera P, Sarkisyan G, Gima L, Boyer JD, Bamias G, Jedlicka P, Veny M, Clark D, et al. (2017) Sphingosine-1-phosphate receptor-1 $\left(\mathrm{S}_{1} \mathrm{P}_{1}\right)$ is 
expressed by lymphocytes, dendritic cells, and endothelium and modulated during inflammatory bowel disease. Mucosal Immunol 10:162-171.

Kuan S, Chapel S, Briggs E, and Tran J (2019) Population pharmacokinetic and pharmacodynamic anlaysis of CC112273 following oral administration of ozanimod in adult subjects (abstract) 10th American Conference on Pharmacometrics (ACoP), ACoP10; 2019 October 20-23; Orlando FL.

Martinborough E, Boehm MF, Yeager AR, Tamiya J, Huang L, Brahmachary E, Moorjani M, Alan G, Brooks JL, and Peach R (2015) inventors. Selective Sphingosine-1 Phosphate Receptor Modulators and Methods of Chiral Synthesis. U.S. patent US2015/0299149.

Miron VE, Jung CG, Kim HJ, Kennedy TE, Soliven B, and Antel JP (2008) FTY720 modulates human oligodendrocyte progenitor process extension and survival. Ann Neurol 63:61-71.

Roffey SJ, Obach RS, Gedge JI, and Smith DA (2007) What is the objective of the mass balance study? A retrospective analysis of data in animal and human excretion studies employing radiolabeled drugs. Drug Metab Rev 39:17-43.

Sandborn W J Feagan BG, Wolf DC, D'Haens G, Vermeire S, Hanauer SB, Ghosh S, Smith H, Cravets M, Frohna PA, Aranda R, Gujrathi S, Olson A (2016) TOUCHSTONE Study Group. Ozanimod Induction and Maintenance Treatment for Ulcerative Colitis. N Eng J Med 374 (18): 1754-1762.

Scott F, Timony G, Brooks J, Peach R, Clemons B, Desale H, Moorjani M, Brahmachary E, Powell R, Dedman H, et al. (2013) Metabolites of RPC1063 contribute to in vivo efficacy. Neurology 80 (Suppl):P05.157.

Scott Fiona L, Clemons B, Brooks J, Brahmachary E, Powell R, Dedman H, Desale HG, Timony GA, Martinborough E, and Rosen H (2016) Ozanimod (RPC1063) is a potent sphingosine- 1phosphate receptor-1 $\left(\mathrm{S}_{1} \mathrm{P}_{1}\right)$ and receptor-5 $\left(\mathrm{S}_{1} \mathrm{P}_{5}\right)$ agonist with autoimmune diseasemodifying activity. British Journal of Pharmacology 173:1778-1792.

Subei AM and Cohen JA (2015) Sphingosine 1-phosphate receptor modulators in multiple sclerosis. CNS Drugs 29:565-575.

Tran JQ, Hartung JP, Olson AD, Mendzelevski B, Timony GA, Boehm MF, Peach RJ, Gujrathi S, and Frohna PA (2018a) Cardiac safety of ozanimod, a novel sphingosine-1-phosphate receptor modulator: results of a thorough QT/QTc study. Clin Pharmacol Drug Dev 7:263-276.
Tran JQ, Hartung JP, Peach RJ, Boehm MF, Rosen H, Smith H, Brooks JL, Timony GA, Olson AD, Gujrathi S, et al. (2017) Results from the first-in-human study with ozanimod, a novel, selective sphingosine-1-phosphate (S1P) receptor modulator. J Clin Pharmacol 57:988-996.

Tran JQ, Hartung JP, Tompkins CA, and Frohna PA (2018b) Effects of high- and low-fat meals on the pharmacokinetics of ozanimod, a novel sphingosine-1-phosphate receptor modulator. Clin Pharmacol Drug Dev 7:634-640.

Tran JQ, Zhang P, Walker S, Ghosh A, Syto M, Wang X, Harris S, and Palmisano M (2020) Multiple-dose pharmacokinetics of ozanimod and its major active metabolites and the pharmacodynamic and pharmacokinetic interactions with pseudoephedrine, a sympathomimetic agent, in healthy subjects. Adv Ther 37:4944-4958.

van Doorn R, Nijland PG, Dekker N, Witte ME, Lopes-Pinheiro MA, van het Hof B, Kooij G, Reijerkerk A, Dijkstra C, van van der Valk P, et al. (2012) Fingolimod attenuates ceramideinduced blood-brain barrier dysfunction in multiple sclerosis by targeting reactive astrocytes Acta Neuropathol 124:397-410.

Yabuki M, Shono F, Nakatsuka I, and Yoshitake A (1993) Novel cleavage of the 1,2,4-oxadiazole ring in rat metabolism of SM-6586, a dihydropyridine calcium antagonist. Drug Metab Dispos 21:1167-1169. Zhang D, Raghavan N, Chen SY, Zhang H, Quan M, Lecureux L, Patrone LM, Lam PY, Bonacorsi SJ, Knabb RM, et al. (2008) Reductive isoxazole ring opening of the anticoagulant razaxaban is the major metabolic clearance pathway in rats and dogs. Drug Metab Dispos 36:303-315.

Zollinger M, Gschwind HP, Jin Y, Sayer C, Zécri F, and Hartmann S (2011) Absorption and disposition of the sphingosine 1-phosphate receptor modulator fingolimod (FTY720) in healthy volunteers: a case of xenobiotic biotransformation following endogenous metabolic pathways Drug Metab Dispos 39:199-207.

Address correspondence to: Dr. Sekhar Surapaneni, Nonclinical Research and Development, Bristol Myers Squibb, 556 Morris Ave., Summit, NJ 07901. E-mail: Sekhar.surapaneni@bms.com 